\title{
Localization of a polymer in random media: Relation to the localization of a quantum particle
}

\author{
Yohannes Shiferaw and Yadin Y. Goldschmidt \\ Department of Physics and Astronomy, \\ University of Pittsburgh, Pittsburgh PA 15260, U.S.A.
}

(November 3, 2018)

\begin{abstract}
In this paper we consider in detail the connection between the problem of a polymer in a random medium and that of a quantum particle in a random potential. We are interested in a system of finite volume where the polymer is known to be localized inside a low minimum of the potential. We show how the end-to-end distance of a polymer which is free to move can be obtained from the density of states of the quantum particle using extreme value statistics. We give a physical interpretation to the recently discovered onestep replica-symmetry-breaking solution for the polymer (Phys. Rev. E61, 1729 (2000)) in terms of the statistics of localized tail states. Numerical solutions of the variational equations for chains of different length are performed and compared with quenched averages computed directly by using the eigenfunctions and eigenenergies of the Schrödinger equation for a particle in a one-dimensional random potential. The quantities investigated are the radius of gyration of a free gaussian chain, its mean square distance from the origin and the end-to-end distance of a tethered chain. The probability distribution for the position of the chain is also investigated. The glassiness of the system
\end{abstract}


is explained and is estimated from the variance of the measured quantities.

PACS number(s): 36.20.Ey, 05.40-a, 75.10.Nr, 64.60.Cn 


\section{INTRODUCTION}

The behavior of polymer chains in random media is a well studied problem [1-8] that has applications in diverse fields. Besides the polymers themselves this problem is directly related to the statistical mechanics of a quantum particle in a random potential [9], the behavior of flux lines in superconductors in the presence of columnar defects [10,11], and the problem of diffusion in a random catalytic environment [4]. It was found in Ref. [2 4. 7] that a very long Gaussian chain, immersed in a random medium with very short range correlations of the disorder, will typically curl up in some small region of low potential energy. The polymer chain is said to be localized and for long chains the end-to-end distance becomes independent of chain length $\left(R^{2} \sim L^{0}\right)$. For short chains the end-to-end distance scales diffusively $\left(R^{2} \sim L\right)$. In a related paper [9], it was found, using the replica approach, that a quantum particle exhibits glassy behavior at low temperatures. The low temperature limit for a quantum particle translates into the long chain limit for polymers. It implies that the free energy landscape of a long chain is typically very complicated and possesses many metastable states.

Recently a new variational solution has been found for gaussian chain embedded in a random $\delta$-correlated potential [7]. This solution, which involve replica-symmetry-breaking (RSB) at the 1-step level, gives rise to the correct behavior of the end-to-end distance of the chain as predicted by the heuristic free-energy estimates of Cates and Ball [3] . It predicts the subtle $\log \mathcal{V}$ dependence of the chain size, which was not present in the variational solution of Edwards and Muthukumar [2] due to the fact that their solution did not contain enough variational parameters and hence did not reflect the translational invariance of the original hamiltonian.

Our aim in this paper is two fold. The first goal is to strengthen and elucidate the connection between the localization of a polymer in a random medium and that of a quantum particle. There is an extensive literature on electron localization that we would like to build bridges into. The second goal is to give further interpretation and a better physical picture

of the recently discovered variational solution for a polymer in a random medium [7]. It 
turns out that these two goals intertwine together by using the properties of the solutions of the Schrödinger equation for a quantum particle in a random environment.

Using the path integral mapping between the partition function of a Gaussian polymer chain and the imaginary time Schrödinger equation, we show that the glassy behavior of the polymer chain can be understood by studying the eigenfunctions of the Schrödinger equation with a random potential. In particular, the phenomenon of Anderson Localization 12,13 is crucial to understanding the glassy phase. We present evidence that shows that polymer localization and glassy behavior can be traced to the dominance of exponentially localized tail states. We also explore various connections between typical glassy behavior, such as non-ergodicity and the existence of many metastable states, to the properties of the eigenfunctions of the Schrödinger equation.

In order to describe the glass phase analytically we utilize the variational replica approach described in [7] and [9]. We further investigate and interpret the transition discovered there between a replica symmetric phase to a 1-step replica symmetry breaking phase at a critical chain length. The 1-step breaking solution describes the localized (glassy) phase of the polymer and corresponds to the dominance of localized tail states. In the long chain limit the lowest tail state in each realization of the disorder will dominate the partition sum. On the other hand, the replica symmetric phase corresponds to the case when a multitude of extended states dominate the partition function. Our results are substantiated by a numerical solution of the problem by solving the imaginary time Schrödinger equation on a lattice in one spatial dimension.

\section{THE MODEL}

The simplest model of a polymer chain in random media is a Gaussian (flexible) chain [14] in a medium of fixed random obstacles [6]. In this paper we do not include a self-avoiding interaction. This model can be described by the Hamiltonian

$$
H=\int_{0}^{L} d u\left[\frac{M}{2}\left(\frac{d \mathbf{R}(u)}{d u}\right)^{2}+\frac{\mu}{2} \mathbf{R}^{2}(u)+V(\mathbf{R}(u))\right],
$$


where $\mathbf{R}(u)$ is the $d$ dimensional position vector of a point on the polymer at arc-length $u(0 \leq u \leq L)$, and where $L$ is the contour length of the chain (number of links). The random medium is described by a random potential $V(\mathbf{R})$ that is taken from a Gaussian distribution that satisfies

$$
\langle V(\mathbf{R})\rangle=0, \quad\left\langle V(\mathbf{R}) V\left(\mathbf{R}^{\prime}\right)\right\rangle=f\left(\left(\mathbf{R}-\mathbf{R}^{\prime}\right)^{2}\right)
$$

The harmonic term in the Hamiltonian is included to mimic the effects of finite volume. This is important to ensure that the model is well defined, since it turns out that certain equilibrium properties of the polymer diverge in the infinite volume limit $(\mu \rightarrow 0)$. The function $f$ characterizes the correlations of the random potential, and will depend on the particular problem at hand. The parameter $M$ is equal to $d /\left(\beta b^{2}\right)$, where $\beta=\left(k_{B} T\right)^{-1}$, and where $b$ is the Khun bond step.

In this paper we will consider Gaussian distributed random media defined by the correlation function

$$
\left.f\left(\left(\mathbf{R}-\mathbf{R}^{\prime}\right)^{2}\right)\right)=\frac{g}{\left(\pi \xi^{2}\right)^{d / 2}} \exp \left(-\left(\mathbf{R}-\mathbf{R}^{\prime}\right)^{2} / \xi^{2}\right)
$$

Here $g$ determines the strength of the disorder and the parameter $\xi$ controls the correlation range of the random media. For $\xi$ large (long range correlations) we expect to recover the exact results presented in Ref. [8]. When $\xi$ is small then we have the more physically relevant case of short range correlated media. In the limit of $\xi \rightarrow 0, f$ approaches a $d$-dimensional $\delta$-function. This was the case studied in Refs. [2:7]. In the present paper we investigate only the case of short-range correlations, i.e. the case of small $\xi$.

Once we have defined the Hamiltonian for any chain configuration $\mathbf{R}(u)$, we can write the partition sum (Green's function) for the set of paths of length $L$ that go from $\mathbf{R}$ to $\mathbf{R}^{\prime}$ as

$$
Z\left(\mathbf{R}, \mathbf{R}^{\prime} ; L\right)=\int_{\mathbf{R}(0)=\mathbf{R}}^{\mathbf{R}(L)=\mathbf{R}^{\prime}}[d \mathbf{R}(u)] \exp (-\beta H)
$$

All the statistical properties of the polymer will depend on the partition sum. For instance, the end-to-end distance (or radius of gyration) of a polymer chain that is free to 
move is given by

$$
\overline{\left\langle\mathbf{R}_{F}^{2}(L)\right\rangle}=\overline{\left(\frac{\int d \mathbf{R} d \mathbf{R}^{\prime}\left(\mathbf{R}-\mathbf{R}^{\prime}\right)^{2} Z\left(\mathbf{R}, \mathbf{R}^{\prime} ; L\right)}{\int d \mathbf{R} d \mathbf{R}^{\prime} Z\left(\mathbf{R}, \mathbf{R}^{\prime} ; L\right)}\right)},
$$

where the overbar stands for the average of the ratio over the realizations of the random potential. This average is referred to as a quenched average, as opposed to an annealed average, where the numerator and denominator are averaged independently. In some previous studies it has been customary to replace the quenched average of certain quantities (and in particular $R_{F}$ ) by the more analytically tractable annealed average. However, this replacement can be justified in the case of Eq. (2.5) only when the system size is strictly infinite, since only in that limit can the polymer sample all of space and find the most favorable potential which will be similar to its environment in the annealed case. The main problem with this approach is that in practice we always deal with finite size systems, and in general it is difficult to assess how big the system size has to be so that the annealed average is a good approximation to the quenched average. In addition, the time it takes the chain to sample a large volume is exceedingly long and unreachable over a reasonable experimental time. In this paper we will always deal with an explicitly finite system (both analytically and numerically) and so we will only compute quenched averages. For very large but finite systems the free chain is said to be localized in the sense that its configuration space is dominated by a single configuration in which the chain is being trapped in a single small neighborhood. The size of the chain in an uncorrelated random potential is given by [3, ]]

$$
R_{F} \propto(g \ln \mathcal{V})^{-1 /(d-4)}
$$

where $\mathcal{V}$ is the volume of the system. The depth of the well entrapping the chain is approximately

$$
V_{\text {min }} \sim(g \ln \mathcal{V})^{2 /(d-4)}
$$

This is the binding energy per monomer. Thus the binding energy of the chain is given by $L V_{\text {min }}$. 
Another quantity of interest is the averaged mean squared displacement of the far end of a polymer with one end that is fixed at the origin. This is a measure of the wandering of a tethered polymer immersed in a random medium. This quantity can be written as

$$
\overline{\left\langle\mathbf{R}_{T}^{2}(L)\right\rangle}=\overline{\left(\frac{\int d \mathbf{R} \mathbf{R}^{2} Z(\mathbf{0}, \mathbf{R} ; L)}{\int d \mathbf{R} Z(\mathbf{0}, \mathbf{R} ; L)}\right)} .
$$

It is important to distinguish between these two quantities since they measure different properties of the polymer chain. Cates and Ball [0] and Nattermann [4] discussed the different behaviors of these quantities for a short-range correlated random potential using heuristic arguments. In a recent paper [8] we have found analytically that the scaling properties of $R_{F}$ and $R_{T}$ are also very different when the disorder has long range quadratic correlations. This is because in the case of a tethered chain, the free end of the chain seeks out favorable regions in the random medium that are typically very far from the origin. When both ends are free then the entire chain will simply curl up in a favorable region in the random medium, and the end-to-end distance will not scale as fast. For the tethered chain the quenched and annealed averages are not expected to coincide even in the infinite volume limit, since a tethered chain can only sample a finite volume of size $L^{d}$, and even for large $L$ the environment near its tail can not be adjusted since it is immobile.

Yet another quantity of interest is

$$
\overline{\left\langle\mathbf{R}_{Q}^{2}(L)\right\rangle}=\overline{\left(\frac{\int d \mathbf{R} \mathbf{R}^{2} Z(\mathbf{R}, \mathbf{R} ; L)}{\int d \mathbf{R} Z(\mathbf{R}, \mathbf{R} ; L)}\right)},
$$

which measures the distance from the origin (of the harmonic potential) to the average (center of mass) position of a free chain (more precisely of a free loop). It is also relevant to the related problem of a quantum particle in a random potential [9,[15. The relation between the polymer and the quantum particle problems arises from the fact that the partition sum of a polymer chain can be mapped to the density matrix of a quantum particle. The mapping [9.16] is given by

$$
\beta \rightarrow 1 / \hbar, L \rightarrow \beta \hbar .
$$

Then $\rho\left(R, R^{\prime} ; \beta\right)=Z\left(R, R^{\prime} ; L=\beta \hbar, \beta=1 / \hbar\right)$ is the density matrix of a quantum particle at inverse temperature $\beta$. Note that the variable $u$ is now interpreted as the Trotter (imaginary) 
time, and $M$ as the mass of the quantum particle. Under this mapping $\overline{\left\langle\mathbf{R}_{Q}^{2}(L)\right\rangle}$ can be interpreted as the average mean squared displacement from the origin of a quantum particle in a random plus harmonic potential centered at the origin.

Finally we would like to remind the reader the definition of the density of states for a quantum particle in a random potential. We first define the quantity $\tilde{\rho}(\beta)$ which is the two-sided Laplace transform of the density of states $\rho(E)$ :

$$
\tilde{\rho}(\beta)=\int_{-\infty}^{\infty} \exp (-\beta E) \rho(E) d E
$$

This function is given by

$$
\tilde{\rho}(\beta)=\lim _{\mathcal{V} \rightarrow \infty} \frac{1}{\mathcal{V}} \int d \mathbf{R} \overline{Z(\mathbf{R}, \mathbf{R} ; \beta)}
$$

We will make use of these quantities in the following sections.

\section{THE PATH INTEGRAL MAPPING}

The partition sum of the polymer chain (2.4) can be mapped to an imaginary time Schrödinger equation. This mapping (see Ref. [16] Eqs. (3.12)-(3.18)) is given by

$$
Z\left(\mathbf{R}, \mathbf{R}^{\prime} ; L\right)=\int_{\mathbf{R}(0)=\mathbf{R}^{\prime}}^{\mathbf{R}(L)=\mathbf{R}}[d \mathbf{R}(u)] \exp (-\beta H[\mathbf{R}(u)])=\left\langle\mathbf{R}|\exp (-\beta L \hat{H})| \mathbf{R}^{\prime}\right\rangle,
$$

where

$$
\hat{H}=-\frac{1}{2 M \beta^{2}} \frac{\partial^{2}}{\partial \hat{\mathbf{R}}^{2}}+\frac{\mu}{2} \hat{\mathbf{R}}^{2}+V(\hat{\mathbf{R}})
$$

So for a given realization of the random potential the polymer partition sum can be expressed as a matrix element of the imaginary-time evolution operator. The matrix elements can be expanded in a complete set of eigenfunctions of the Hamiltonian operator to yield

$$
\left\langle\mathbf{R}|\exp (-\beta L \hat{H})| \mathbf{R}^{\prime}\right\rangle=\sum_{m=0}^{\infty} \exp \left(-\beta L E_{m}\right) \Phi_{m}(\mathbf{R}) \Phi_{m}^{*}\left(\mathbf{R}^{\prime}\right),
$$

where

$$
\hat{H} \Phi_{m}(\mathbf{R})=E_{m} \Phi_{m}(\mathbf{R})
$$


It is clear from the above relation that all properties of the polymer chain will depend on the eigenvalues and the eigenfunctions of the Hamiltonian operator $\hat{H}$. For the problem at hand we will have to understand these properties for the case when the potential $V(\mathbf{R})$ is random and with the correlations given in Eq. (2.3) with a small $\xi$.

The Schrödinger equation with a random potential is a well known problem that has been intensely studied for a long time [12,13, 17, 25]. The main property that we will use is that when $V(\mathbf{R})$ has short range correlations (i.e. correlation length is shorter than any other length scale in the problem), and if the system size is infinite, then in any dimension all eigenstates with energy bellow a critical energy $E_{M}$ ( refered to as the mobility edge) are exponentially localized in the form

$$
\Phi_{m}(\mathbf{R}) \sim \exp \left(-\left|\mathbf{R}-\mathbf{R}_{m}\right| / \ell_{m}\right)
$$

Here $\mathbf{R}_{m}$ is the localization center of the $m^{\text {th }}$ state, and $\ell_{m}$ is the localization length of that state. The localization length satisfies

$$
1 / \ell_{m}=\beta \sqrt{2 M\left|E_{m}\right|}
$$

for $E_{m} \ll 0$, i.e. deep in the tail region. Intuitively, it is easy to verify these last two relations for the solution of the Schrödinger equation for a particle in a one dimensional, non-random, attractive $\delta$-function potential. This can be thought to represent a local minimum of the random potential. In $d$-dimensions one can similarly consider a potential of the form $-v \delta(r)$ and the lowest energy solution of the radial equation (with zero angular momentum) also satisfies these relations. For $E>E_{M}$ extended states exist when $d>2$. For $d=1,2$ there is no mobility edge and all states are exponentially localized. The states with energies $E>E_{M}$ are called extended since they are no longer localized but are spread over a finite fraction of the system. Also, it is known that the eigenvalues of the localized states are discrete, while the eigenvalues of the extended states form a continuum.

For finite system size, or if $\mu \neq 0$ in the Hamiltonian given in Eq. (3.2), the above discussion has to be modified. First, the eigenfunctions are always discrete in any dimensions. But even in one dimension as the energy increases the width of the localized states eventually 
becomes comparable to the system size and thus a localized particle of that energy can go from one end of the sample to the other. Thus the distinction between localized and extended states becomes blurred for a finite system at energies much above the ground state. Nevertheless, there will still be a qualitative difference between the low energy tail states and the higher energy states with large localization lengths. For a finite system (or when $\mu \neq 0$ ) and for any given realization of the random potential there will always be a lowest energy state which is by definition the ground state for that realization. If the volume is large (or $\mu$ small) it will correspond to one of the deep tail states of the infinite system.

In order to study the effect of the eigenfunctions on the physical properties of the polymer chain, we simply apply Eq. (3.3). It is clear that when $L$ (or $\beta$ ) is large the partition sum is dominated by contributions due to the low energy localized states, while if $L$ is small then most of the contributions will come from the continuum of extended states. As $L$ is slowly increased then fewer and fewer localized states contribute to the sum, until we attain ground state dominance at some very large $L$. For simplicity let us concentrate on the diagonal elements of the evolution operator. Then

$$
Z(\mathbf{R}, \mathbf{R} ; L)=\sum_{m} \exp \left(-\beta L E_{m}\right)\left|\Phi_{m}(\mathbf{R})\right|^{2},
$$

which is proportional to the probability of finding a loop of length $L$ passing through the point $\mathbf{R}$. Since the eigenstates for low energies are localized then for large $L$ we can write

$$
Z(\mathbf{R}, \mathbf{R} ; L) \sim \sum_{m} \exp \left(-\beta L E_{m}\right) \exp \left(-2\left|\mathbf{R}-\mathbf{R}_{m}\right| / \ell_{m}\right) .
$$

This implies that the probability of finding a long loop passing through $\mathbf{R}$ is concentrated around the localization centers of the low energy eigenstates. Consequently, a very long polymer chain will most likely be found at the ground state localization center. When $L$ is small then the partition sum will be dominated by the extended states, and so the probability of finding a loop at $\mathbf{R}$ should be fairly uniform throughout the system. In a later section we will analyze in detail the evolution of the partition sum with $L$ by solving the Schrödinger equation on a lattice in $d=1$. 
All the physical properties of the polymer chain can be expressed in terms of the eigenstates of Schrödinger equation. For instance we can write the end-to-end distance for a given realization of the random potential as

$$
\left\langle\mathbf{R}_{F}^{2}(L)\right\rangle_{V}=\frac{2 \sum_{m}\left(a_{m} \int d \mathbf{R} \mathbf{R}^{2} \Phi_{m}^{*}(\mathbf{R})-\left|\int d \mathbf{R} \mathbf{R} \Phi_{m}(\mathbf{R})\right|^{2}\right) \exp \left(-\beta L E_{m}\right)}{\sum_{m}\left|a_{m}\right|^{2} \exp \left(-\beta L E_{m}\right)},
$$

where $a_{m}=\int d \mathbf{R} \Phi_{m}(\mathbf{R})$, and where $\langle\cdot\rangle_{V}$ refers to a configurational average for the case of a fixed realization of random potential. When $L$ is large enough so that $\left(E_{1}-E_{g s}\right) L>>1$, where $E_{1}$ is the eigenvalue of the first excited state, then only the ground state contributes. In this case we have

$$
\left\langle\mathbf{R}_{F}^{2}(L)\right\rangle_{V}=2 \frac{\int d \mathbf{R} \mathbf{R}^{2} \Phi_{g s}(\mathbf{R})}{\int d \mathbf{R} \Phi_{g s}(\mathbf{R})}-2\left(\frac{\int d \mathbf{R} \mathbf{R} \Phi_{g s}(\mathbf{R})}{\int d \mathbf{R} \Phi_{g s}(\mathbf{R})}\right)^{2},
$$

where $\Phi_{g s}(\mathbf{R})$ is the ground state eigenfunction. It can be shown that the ground state wave function is positive definite and so in the large $L$ limit $\left\langle\mathbf{R}_{F}^{2}(L)\right\rangle^{1 / 2}$ can be interpreted as the width of the ground state eigenfunction. Assuming the ground state has the form given in Eq. (3.5), we can write $\left\langle\mathbf{R}_{F}^{2}(L)\right\rangle_{V}=2 d(d+1) \ell_{g s}^{2}$, where $\ell_{g s}=\ell_{0}$ is the localization length of the ground state. Upon averaging over all realizations of the random potential we get that $\overline{\left\langle\mathbf{R}_{F}^{2}(L)\right\rangle}=2 d(d+1) \overline{\ell_{g s}^{2}}$, and so the quenched average of the end-to-end distance, in the long chain limit, is proportional to the square of the average localization length of the ground state eigenfunction. We see that for $\mu \rightarrow 0$ the average is taken over the tail states of the Schrödinger equation.

When one end of the polymer is tethered to the origin then the end-to-end distance can be expanded in eigenfunctions to yield

$$
\left\langle\mathbf{R}_{T}^{2}(L)\right\rangle_{V}=\frac{\sum_{m} \int d \mathbf{R} \mathbf{R}^{2} \Phi_{m}^{*}(\mathbf{R}) \Phi_{m}(\mathbf{0}) \exp \left(-\beta L E_{m}\right)}{\sum_{m} \int d \mathbf{R} \Phi_{m}^{*}(\mathbf{R}) \Phi_{m}(\mathbf{0}) \exp \left(-\beta L E_{m}\right)} .
$$

The presence of the term $\Phi_{m}(\mathbf{0})$ is crucial since if the eigenstates are localized then $\Phi_{m}(\mathbf{0}) \sim$ $\exp \left(-\left|\mathbf{R}_{m}\right| / \ell_{m}\right)$, which means that eigenstates localized far away from the origin may not contribute to the sum even if they may have very low energies. When $L$ is not very large the extended states will dominate the sums in Eq. (3.11) and the behavior of the endto-end distance should be diffusive $\left(\left\langle\mathbf{R}_{T}^{2}(L)\right\rangle_{V} \sim L\right)$. When $L$ is large, but before the 
onset of ground state dominance, the sums in Eq. (3.11) will be dominated by localized eigenstates that are centered close to the origin. The free end of the polymer chain will hop between localization centers as $L$ increases. This behavior has been investigated using Flory arguments in Ref. [4, 5] and both authors found a weakly sub-ballistic $\left(\overline{\left\langle\mathbf{R}_{T}^{2}(L)\right\rangle} \sim\right.$ $L^{2} / \log (L)^{\gamma}$ ) behavior of the mean squared displacement. For a finite system and when $L$ is large enough the ground state will always dominate and we get $\left\langle\mathbf{R}_{T}^{2}(L)\right\rangle_{V}=d(d+1) \ell_{g s}^{2}+\mathbf{R}_{g s}^{2}$. Since the distance from the ground state to the origin will typically be much larger than the localization length, we find upon averaging that $\overline{\left\langle\mathbf{R}_{T}^{2}(L)\right\rangle} \approx \overline{\mathbf{R}_{g s}^{2}}$, and so the quenched average of the end-to-end distance simply converges to the distance to the localization center of the ground state. This distance is of the order of the size of the system. Thus the interesting sub-ballistic $L$-dependence of $\overline{\left\langle\mathbf{R}_{T}^{2}(L)\right\rangle}$ arises from the contribution of excited states and not from ground state dominance as for the case of $\overline{\left\langle\mathbf{R}_{F}^{2}(L)\right\rangle}$.

The mean squared displacement defined in Eq. (2.9) can also be expanded in eigenfunctions as

$$
\left\langle\mathbf{R}_{Q}^{2}(L)\right\rangle_{V}=\frac{\sum_{m} \int d \mathbf{R} \mathbf{R}^{2}\left|\Phi_{m}(\mathbf{R})\right|^{2} \exp \left(-\beta L E_{m}\right)}{\sum_{m} \exp \left(-\beta L E_{m}\right)} .
$$

In the large $L$ limit we find that $\left\langle\mathbf{R}_{Q}^{2}(L)\right\rangle_{V}=d(d+1) \ell_{g s}^{2} / 4+\mathbf{R}_{g s}^{2}$. This is very similar to the case discussed in the previous paragraph and we find upon averaging that $\overline{\left\langle\mathbf{R}_{Q}^{2}(L)\right\rangle} \approx \overline{\mathbf{R}_{g s}^{2}}$. Which implies that $\overline{\left\langle\mathbf{R}_{Q}^{2}(L)\right\rangle}$ becomes independent of $L$. When $L$ is small then the extended states dominate and we expect a behavior as if there is no random medium. For a polymer confined by a quadratic potential we find that $\overline{\left\langle\mathbf{R}_{Q}^{2}(L)\right\rangle}=d(\beta \mu L)^{-1}$.

The relationship between the eigenstates of the Schrödinger equation and the partition sum of the polymer chain can also be used to understand the behavior of the sample-tosample variations of various physical properties of the chain. The sample-to-sample variations are important in order to assess whether the average (over many samples) of a physical quantity is an accurate measure of that quantity for a typical sample. For instance, the

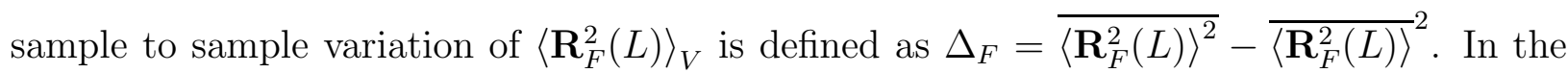
same way we can define the sample-to-sample variation $\Delta_{T}={\overline{\left\langle\mathbf{R}_{T}^{2}(L)\right\rangle^{2}}}-{\overline{\left\langle\mathbf{R}_{T}^{2}(L)\right\rangle}}^{2}$ and

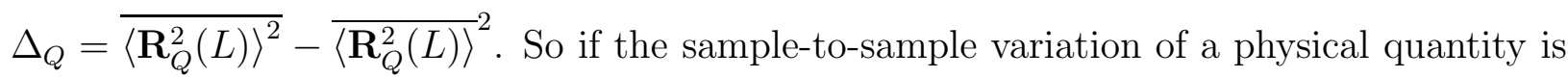


large then that implies that the probability distribution for that quantity is highly skewed and strongly dependent on the random sample. Systems which exhibit such behavior are said to have glassy properties. For simplicity if we assume ground state dominance ( $L$ very large) then $\Delta_{F}$ is just a measure of the sample-to-sample variation of the localization length of the ground state. Also $\Delta_{T}$ and $\Delta_{Q}$ are both a measure of the sample-to-sample variation of the distance from the origin to the ground state localization center. The crucial point here is that the sample-to-sample variations are dependent on which eigenstates dominate the partition sum, and so there should be a connection between glassy properties and the behavior of the eigenstates.

\section{THE REPLICA VARIATIONAL APPROACH}

In this section we review the replica approach that was used in Refs. [7] and [9] to compute quenched averages of various physical properties of the polymer chain. Our goal is to give an interpretation for this formalism in terms of the localized states picture of the corresponding Schrödinger equation.

In order to compute the quenched average over the random potential we apply the replica method. We first introduce $n$-copies of the system and average over the random potential to get

$$
\begin{array}{r}
Z_{n}\left(\left\{\mathbf{R}_{a}\right\},\left\{\mathbf{R}_{a}^{\prime}\right\} ; L\right)=\overline{Z\left(\mathbf{R}_{1}, \mathbf{R}_{1}^{\prime} ; L\right) \cdots Z\left(\mathbf{R}_{n}, \mathbf{R}_{n}^{\prime} ; L\right)} \\
=\int_{\mathbf{R}_{a}(0)=\mathbf{R}_{a}}^{\mathbf{R}_{a}(L)=\mathbf{R}_{a}^{\prime}} \prod_{a=1}^{n}\left[d \mathbf{R}_{a}\right] \exp \left(-\beta H_{n}\right)
\end{array}
$$

where

$$
\begin{gathered}
H_{n}=\frac{1}{2} \int_{0}^{L} d u \sum_{a}\left[M\left(\frac{d \mathbf{R}_{a}(u)}{d u}\right)^{2}+\mu \mathbf{R}_{a}^{2}(u)\right] \\
-\frac{\beta}{2} \int_{0}^{L} d u \int_{0}^{L} d u^{\prime} \sum_{a b} f\left(\left(\mathbf{R}_{a}(u)-\mathbf{R}_{b}\left(u^{\prime}\right)\right)^{2}\right) .
\end{gathered}
$$

The averaged equilibrium properties of the polymer can now be written in terms of the

replicated partition sum $Z_{n}\left(\left\{\mathbf{R}_{a}\right\},\left\{\mathbf{R}_{a}\right\} ; L\right)$. For instance, the mean squared end-to-end 
distance defined in Eq. (2.5) can be written in as

$$
\overline{\left\langle\mathbf{R}_{F}^{2}(L)\right\rangle}=\lim _{n \rightarrow 0} \frac{\int \prod d \mathbf{R}_{a} \prod d \mathbf{R}_{a}^{\prime}\left(\mathbf{R}_{1}-\mathbf{R}_{1}^{\prime}\right)^{2} Z_{n}\left(\left\{\mathbf{R}_{a}\right\},\left\{\mathbf{R}_{a}^{\prime}\right\} ; L\right)}{\int \prod d \mathbf{R}_{a} \prod d \mathbf{R}_{a}^{\prime} Z_{n}\left(\left\{\mathbf{R}_{a}\right\},\left\{\mathbf{R}_{a}^{\prime}\right\} ; L\right)} .
$$

Thus, the averaged equilibrium properties of the polymer can be extracted from an $n$-body problem by taking the $n \rightarrow 0$ limit at the end. This limit has to be taken with care, by solving the problem analytically for general $n$, before taking the limit of $n \rightarrow 0$. Unfortunately the replicated partition sum cannot be evaluated analytically and a variational approach has been used in Refs. [7,9] to make further progress. The procedure is to follow the work of Feynman [16] and others [19,20] and model $H_{n}$ by a solvable trial Hamiltonian $h_{n}$ which is determined by the stationarity of the variational free energy

$$
n\langle F\rangle=\left\langle H_{n}-h_{n}\right\rangle_{h_{n}}-\frac{1}{\beta} \ln \int\left[d \mathbf{R}_{1}\right] \cdots\left[d \mathbf{R}_{n}\right] \exp \left(-\beta h_{n}\right)
$$

Note that the variational free energy also depends on the boundary conditions on the polymer chain. If we are interested in the case when one end is fixed then the partition sum should be over paths with one end fixed. In this case the path integrals should be evaluated using $\int d \mathbf{R}_{i} \int_{\mathbf{R}_{i}(0)=\mathbf{0}}^{\mathbf{R}_{i}(L)=\mathbf{R}_{i}}\left[d \mathbf{R}_{i}\right]$. When both ends are free one should use instead $\int d \mathbf{R}_{i} d \mathbf{R}_{i}^{\prime} \int_{\mathbf{R}_{i}(0)=\mathbf{R}_{i}^{\prime}}^{\mathbf{R}_{i}(L)=\mathbf{R}_{i}}\left[d \mathbf{R}_{i}\right]$. Alternatively, one can evaluate the partition sum with $\int d \mathbf{R}_{i} \int_{\mathbf{R}_{i}(0)=\mathbf{R}_{i}}^{\mathbf{R}_{i}(L)=\mathbf{R}_{i}}\left[d \mathbf{R}_{i}\right]$, which yields the free energy of a polymer loop of contour length $L$. The parameters that minimize the variational free energy for this case should be the same as for the case when both ends are free since in both cases the polymer loop can be anywhere in the system. These are the boundary conditions that were used in Ref. [7], where only the polymer chain that is free to move was investigated.

The quadratic trial Hamiltonian has been parametrized [7] by

$$
\begin{gathered}
h_{n}=\frac{1}{2} \int_{0}^{L} d u \sum_{a}\left[M\left(\frac{d \mathbf{R}_{a}(u)}{d u}\right)^{2}+\mu \mathbf{R}_{a}^{2}(u)\right] \\
-\frac{1}{4 L} \int_{0}^{L} d u \int_{0}^{L} d u^{\prime} \sum_{a b} p_{a b}\left(\mathbf{R}_{a}(u)-\mathbf{R}_{b}\left(u^{\prime}\right)\right)^{2}
\end{gathered}
$$

where the matrix elements $p_{a b}$ are the variational parameters. The physical motivation for this ansatz is that the replica-replica interaction in the original Hamiltonian is modeled by 
a quadratic interaction which can be different for different replica pairs. Also, as noticed in [7], the quadratic interaction has the same translational invariance as the interaction term in the original Hamiltonian. The case when the replica-replica interactions are the same $\left(p_{a b}=\right.$ constant $)$ corresponds to the case of long range quadratic correlations which was solved exactly in [8]. If we expand and simplify the quadratic interaction we can rewrite the trial Hamiltonian as

$$
\begin{aligned}
h_{n}= & \frac{1}{2} \int_{0}^{L} d u \sum_{a}\left[M\left(\frac{d \mathbf{R}_{a}(u)}{d u}\right)^{2}+\lambda \mathbf{R}_{a}^{2}(u)\right] \\
& +\frac{1}{2 L} \int_{0}^{L} d u \int_{0}^{L} d u^{\prime} \sum_{a b} p_{a b} \mathbf{R}_{a}(u) \cdot \mathbf{R}_{b}\left(u^{\prime}\right),
\end{aligned}
$$

where $\lambda=\mu-\sum_{b} p_{a b}$. Here, $\lambda$ is assumed to be independent of the replica index $a$, as is the case if $p$ is a hierarchical matrix. In Ref. [7] $\lambda$ was treated as an independent variational parameter, and the condition $\lambda=\mu-\sum_{b} p_{a b}$ emerged automatically as a result of the translational invariance of the disorder dependent term in the original hamiltonian $H_{n}$.

In the 1-step RSB scheme, the matrix $p_{a b}$ can be parametrized as $(\tilde{p}, p(x))$ with

$$
p(x)= \begin{cases}-s_{0} & 0<x<x_{c} \\ -s_{1} & x_{c}<x<1\end{cases}
$$

and where $x$ is Parisi's replica index. In [7] $\tilde{p}$ has been denoted by $\lambda-\lambda_{1}$. Thus, five variational parameters were used: $\lambda, \lambda_{1}, s_{0}, s_{1}$ and $x_{c}$. The variational free energy was expressed as a function of these variational parameters. Taking the partial derivative with respect to the variational parameters and equating them to zero one gets five non-linear equations which could be solved analytically when $L$ was large and $\mu$ was small [7]. In this paper we started from the same free energy and took its partial derivatives without simplifying the expressions for large $L$. As mentioned above because of the consequences of translational invariance we could reduce the number of parameters and equations to four, since

$$
\lambda_{1}+x_{c} s_{0}+\left(1-x_{c}\right) s_{1}=\mu
$$


We proceeded to solve the equations of stationarity numerically using a standard iterative method 22]. Once the variational parameters have been found we can obtain expressions for various physical quantities. For instance, we find that

$$
\overline{\left\langle\mathbf{R}_{F}^{2}(L)\right\rangle}=\frac{2 d}{\beta \sqrt{M \lambda}} \frac{\sinh \left(\sqrt{\frac{\lambda}{M}} L\right)}{\left(\cosh \left(\sqrt{\frac{\lambda}{M}} L\right)+1\right)}
$$

and also that

$$
\overline{\left\langle\mathbf{R}_{Q}^{2}(L)\right\rangle}=\frac{d}{\beta L}\left(\frac{1}{\mu}-\frac{1}{\lambda}+\frac{s_{0}}{\mu^{2}}+\frac{\left(1-x_{c}\right) \Sigma}{x_{c} \mu(\mu+\Sigma)}\right)+\frac{d}{2 \beta \sqrt{M \lambda}} \operatorname{coth}\left(\sqrt{\frac{\lambda}{M}} \frac{L}{2}\right)
$$

where we have put $\Sigma=x_{c}\left(s_{1}-s_{0}\right)$. Details of the numerical results will be given in the next section. For large $L$ and small $\mu$ it can be seen using the results obtained in [7] that to leading order

$$
\overline{\left\langle\mathbf{R}_{F}^{2}(L)\right\rangle}=\frac{2 d}{\beta \sqrt{M \lambda}},
$$

and

$$
\overline{\left\langle\mathbf{R}_{Q}^{2}(L)\right\rangle}=\frac{1}{\beta \mu L x_{c}}
$$

with

$$
\lambda=\frac{d^{4 /(4-d)}}{(2 \pi)^{2 d /(4-d)}}\left(\beta^{2} M\right)^{(4+d) /(4-d)}(g|\ln \mu|)^{4 /(4-d)}
$$

and

$$
x_{c}=\frac{1}{L}\left(\frac{d^{d-2}}{(2 \pi)^{d}} g^{2} \beta^{d+4} M^{d}|\ln \mu|^{d-2}\right)^{-1 /(4-d)} .
$$

\section{NUMERICAL PROCEDURE}

We check the validity of the analytic solution by numerically computing the quenched average of various physical properties of the polymer. This is computationally intensive because all quantities will have to be averaged over many realizations of the random potential. In this paper we will only concentrate on the case $d=1$. Although this does not correspond 
to a physical polymer $(d=3)$ we will still be able to check the validity of our analytical results for the special case $d=1$.

We evaluate numerically the right hand side of Eq. (3.3) by solving the Schrödinger equation on a one dimensional lattice of $N$ sites [22]. The lattice Hamiltonian is an $N \times N$ matrix with matrix elements given by

$$
H_{i j}=-\frac{1}{2 M \beta^{2} \Delta^{2}}\left(\delta_{i, j+1}+\delta_{i+1, j}\right)+\left(\frac{\mu}{2} \Delta^{2}(i-N / 2)^{2}+V(i)\right) \delta_{i, j}
$$

where the lattice spacing is $\Delta=S / N$, and where $S$ is the system size. Since we are interested in the continuum limit $\Delta$ will be kept small. Note that the index $i$ corresponds to the position $R_{i}=\Delta i$. We impose hard wall boundary conditions at the end of the lattice. The eigenvalues and eigenvectors can now be found directly by diagonalizing the matrix using a standard numerical routine [22]. Once these are known we can construct the partition sum at any value of $L$ using Eq. (3.3). The partition sum can then be used to compute the quantity of interest, such as $\left\langle\mathbf{R}_{F}^{2}(L)\right\rangle_{V}$. We repeat this procedure a large number of times and average the results to get a numerical approximation to $\overline{\left\langle\mathbf{R}_{F}^{2}(L)\right\rangle}$.

The correlated Gaussian random potential described by Eq. (2.3) is modeled by a sequence of $N$ numbers $\left\{V_{\xi}(i)\right\}_{i=1, ., N}$ that obey $\left\langle V_{\xi}(i) V_{\xi}(i+l)\right\rangle \propto \exp \left(-\Delta^{2} l^{2} / \xi^{2}\right)$. These numbers are then placed on a lattice of $N$ sites in the given order. To generate such numbers we use an established method for generating correlated random numbers. The details of this method are described in Ref. [8].

\section{RESULTS AND DISCUSSION}

\section{A. Numerical and Analytical results for $\overline{\left\langle\mathbf{R}_{F}^{2}(L)\right\rangle}$ and $\overline{\left\langle\mathbf{R}_{Q}^{2}(L)\right\rangle}$}

Using the method described in the previous section we compute $\overline{\left\langle\mathbf{R}_{F}^{2}(L)\right\rangle}$ on a lattice of size $N=200(\mathrm{~S}=40)$. Here, we concentrate on the case where the random potential has very short range correlations. We generate random potential samples with correlation length $\xi=1 / \sqrt{2}$. In Fig. [ 1 we show a typical sample of 200 numbers from such a distribution. 


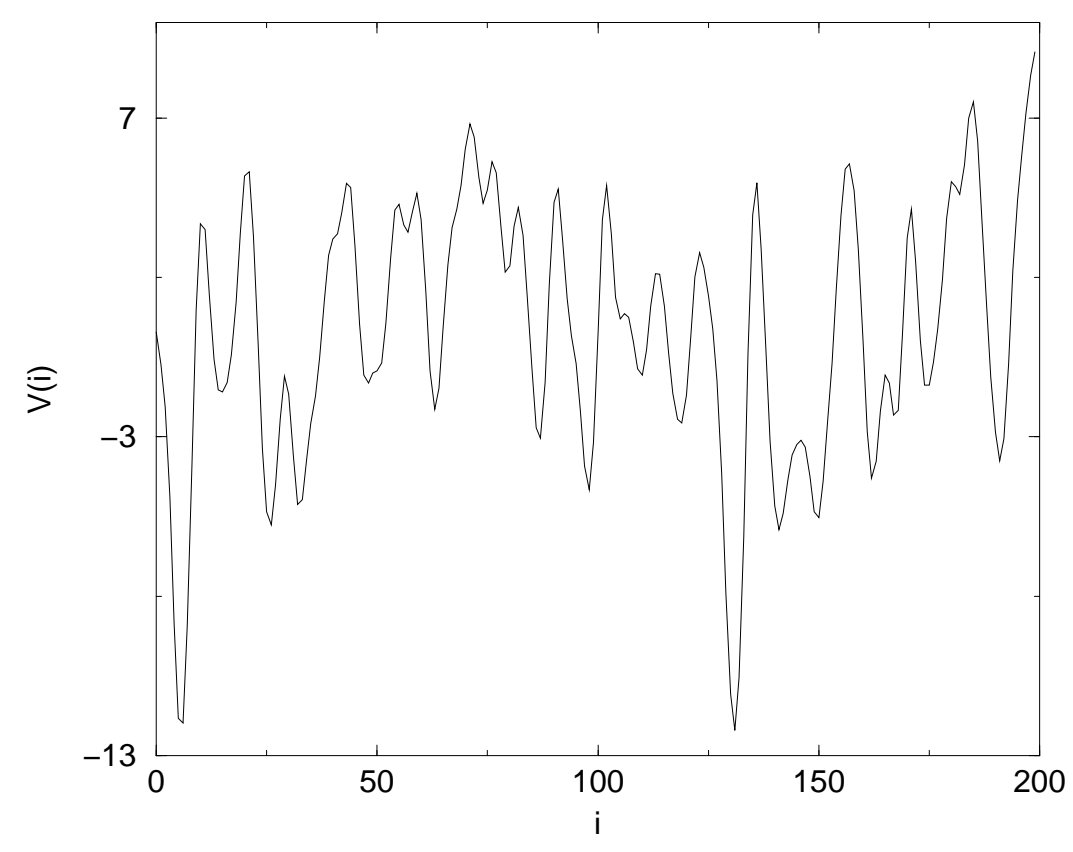

FIG. 1. A typical random sample with $\xi=1 / \sqrt{2}$

Notice that the short range correlation leads to a rugged random potential landscape. The average mean squared displacement $\overline{\left\langle\mathbf{R}_{F}^{2}(L)\right\rangle}$ is computed by averaging over 10000 samples, and the error is estimated by computing the standard deviation of 10 sets of 1000 samples.

We were able to find numerical solutions to the non-linear stationarity equations. We found that for a given set of parameters there is a chain length $L_{c}$ (which depends on the strength of the disorder) such that for $0<L<L_{c}$ there is only a replica symmetric solution. This is the case when the variational parameters satisfy $x_{c}=1$ and $s_{0}=s_{1}$. For $L>L_{c}$ there is still a replica symmetric solution but we also find an additional replica symmetry breaking solution. So in this regime we find an additional solution such that $0<x_{c}<1$ and $s_{0} \neq s_{1}$. In order to decide which solution correctly describes the physics in that regime we compare their respective predictions to the lattice computation of $\overline{\left\langle\mathbf{R}_{F}^{2}(L)\right\rangle}$ and $\overline{\left\langle\mathbf{R}_{Q}^{2}(L)\right\rangle}$.

In Fig. 2 we plot the mean squared displacement $\overline{\left\langle\mathbf{R}_{F}^{2}(L)\right\rangle}$ vs. $L$ for a given set of parameters. We plot this quantity using the lattice result, and also using the two predictions of the variational method. Note that in the labels of the plots the average over the disorder is denoted by a second set of brackets rather than an overbar. For $L$ below $L_{c} \approx 0.73$ there is only a RS solution which is very close to the lattice prediction. For $L$ greater than $L_{c}$ 


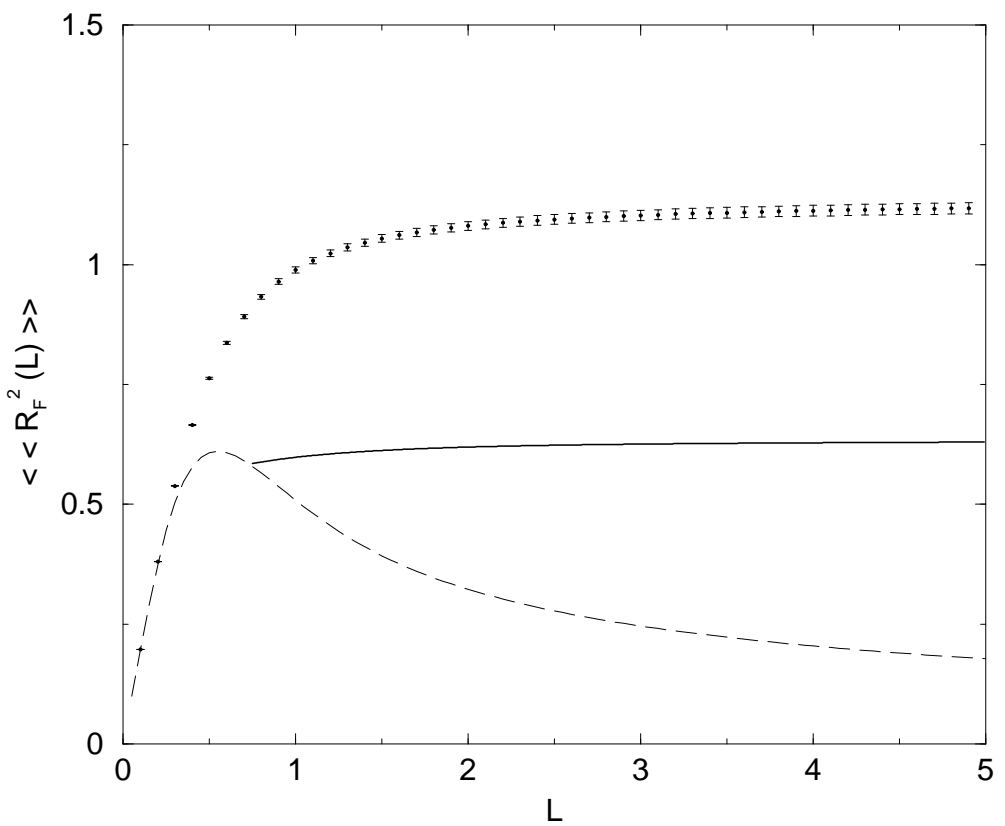

FIG. 2. Plot of $\overline{\left\langle\mathbf{R}_{F}^{2}(L)\right\rangle}$ vs. $L$. The parameters are $M=1 / 2, g=25, \beta=1, \xi=1 / \sqrt{2}$, and $\mu=0.01$. The dotted line is generated by averaging over 10000 samples on a lattice of size $\mathrm{S}=40$ with $\Delta=0.2$. The error bars are found by computing the standard deviation of 10 sets of 1000 samples. The dashed line is the RS solution, and the solid line is the RSB solution. 
the RS and RSB solutions are different and it is clear that the RSB solution is closer to the lattice result. We can see that the end-to-end distance saturates at a constant value as $L$ increases. This behavior is correctly predicted by the RSB solution but not by the RS solution.

We now turn our attention to the quantity $\overline{\left\langle\mathbf{R}_{Q}^{2}(L)\right\rangle}$. In Fig. 3 we plot $\overline{\left\langle\mathbf{R}_{Q}^{2}(L)\right\rangle}$ vs. $L$ using the lattice computation and also using the RS and RSB solutions. We can clearly see that when the RS solution differs from the RSB solution, the RSB prediction is closer to the lattice prediction. Again, the quantity $\overline{\left\langle\mathbf{R}_{Q}^{2}(L)\right\rangle}$ becomes constant for large $L$ and this is correctly predicted by the RSB solution. For this quantity similar results were obtained previously in Refs. [9] and [15].

It is clear from Fig. 2 and 3 that the variational method with the quadratic ansatz in Eq. (4.5) is quite effective in describing the physical properties of the polymer chain. The features predicted by the lattice computation are consistent with a RS solution for chain lengths shorter than $L_{c}$ and a RSB solution for chains longer than $L_{c}$. In a later section we will explore the physical interpretation of the variational solution and show that it is indeed consistent with the physics of the problem.

\section{B. Localized eigenstates and glassy behavior}

In this section we explore, using the lattice computation, the connection between the eigenstates of the Schrödinger equation and the physical properties of the polymer chain. We focus on the probability distribution defined as $P(\mathbf{R}, L)=Z(\mathbf{R}, \mathbf{R}, L) / \int Z(\mathbf{R}, \mathbf{R}, L) d \mathbf{R}$ which can be interpreted as the probability of finding a closed polymer chain of length $L$ which passes through the point $\mathbf{R}$ (for a given realization of the random potential). We consider this probability distribution since it gives the most direct connection between the chain properties and the eigenfunctions of the Schrödinger equation. In Fig. 田 we plot $P(R, L)$ vs. $R$ for four different chain lengths. We also include a plot of the random potential sample that is used. We can see clearly that the probability distribution evolves from a flat distribution to distributions that are sharply peaked at various locations in the 


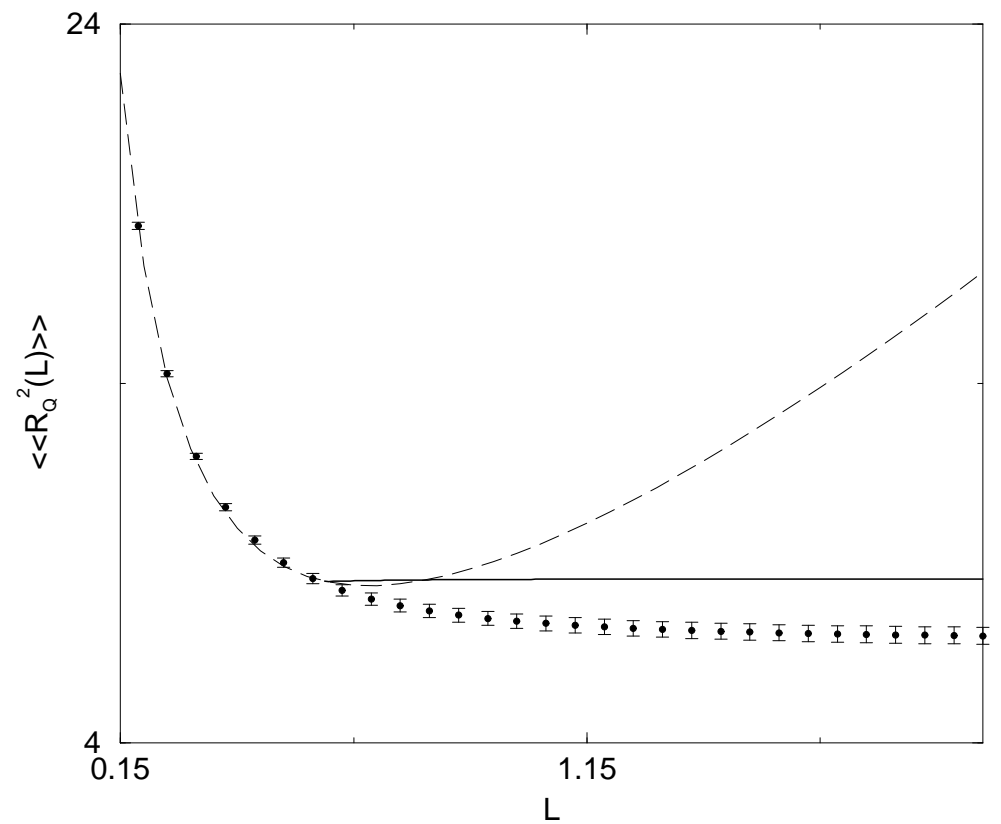

FIG. 3. Plot of $\overline{\left\langle\mathbf{R}_{Q}^{2}(L)\right\rangle}$ vs. L. The parameters are the same as those of Fig. 目 except that here we use $\mu=0.3$. The dotted line is generated by averaging over 10000 samples. The error bars are found by computing the standard deviation of 10 sets of 1000 samples. The dashed line is the RS solution, and the solid line is the RSB solution.

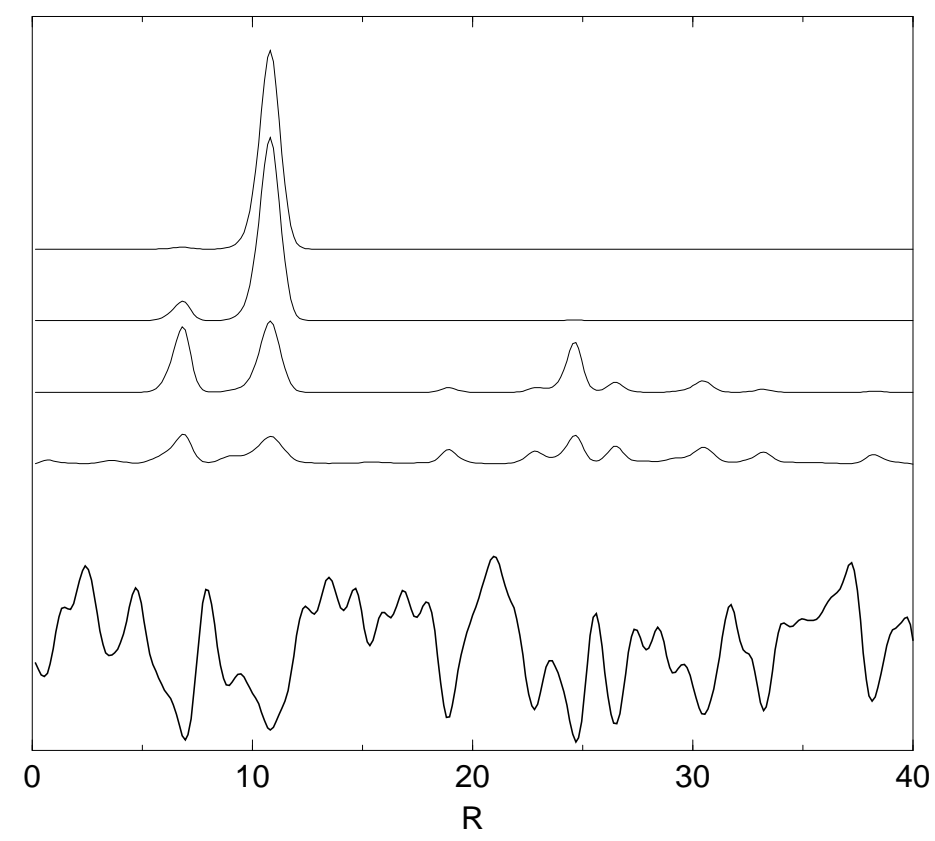

FIG. 4. Plot of $P(R, L)$ vs $R$ for four values of $L$. The bottom most curve is the random potential sample that is used. From bottom to top we use $L=.3,1,10,20$. The parameters are the same as those used for Fig. 目. 


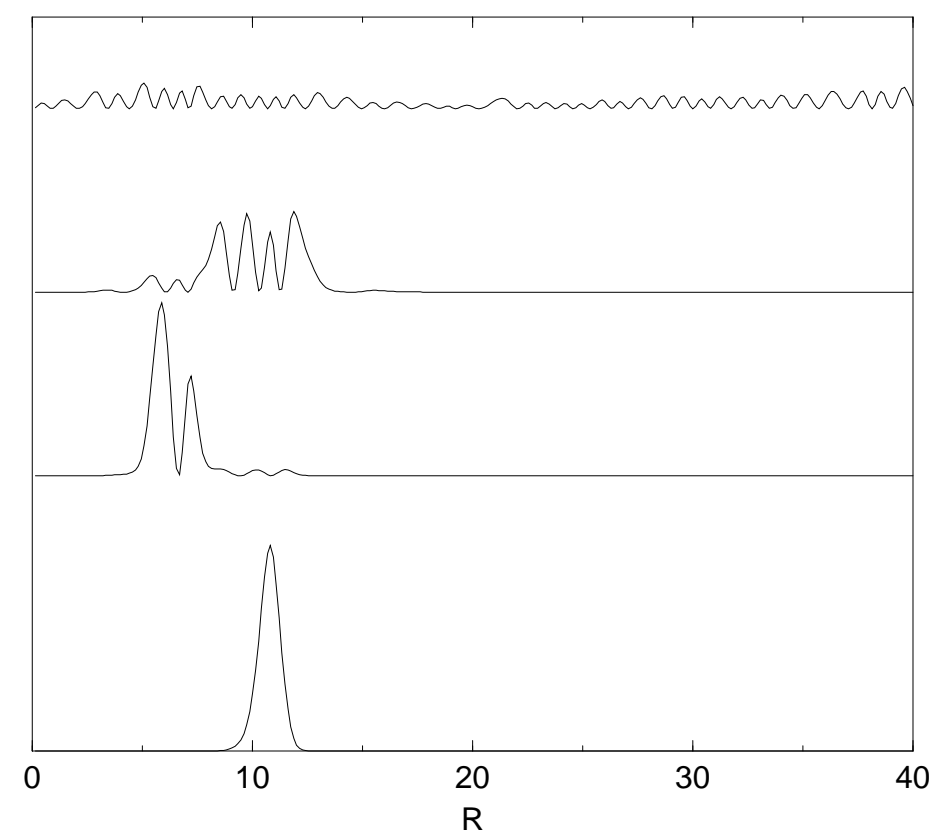

FIG. 5. Plot of $\Phi_{m}^{2}(R)$ vs. $R$. From bottom up we plot the eigenfunctions with $m=0,10,19,39$. We use a lattice of size $\mathrm{S}=40$ with $\Delta=0.133$. All other parameters are the same as that of Fig. 2. sample. Also, an important feature is that the number of peaks decreases with increasing $L$ until finally there is only one peak. This implies that longer chains have a tendency to be found in a few favorable regions in the sample, while short chains can be found with equal probability almost anywhere in the sample. Another observation is that the peaks in the distribution, for the cases $L=1,10,20$, are concentrated around the valleys of the random landscape. This shows that the chain is more likely to be found in regions of low average potential. As can be seen the width of the well is also important.

The results of the previous section can be better understood by studying the properties of the eigenfunctions of the Schrödinger equation in a random potential. Using the eigenfunction expansion in Eq. (3.7) we can see that $P(\mathbf{R}, L)$ is just a sum of the eigenfunctions squared weighted with the Boltzman factor $e^{-\beta L E_{m}}$. So the shape of $P(\mathbf{R}, L)$ is dependent on which eigenstates have the dominant weight at a given chain length $L$. In Fig. 5 we plot a representative sample of the eigenfunctions for a typical random potential sample. In order to get a more general picture of the structure of the eigenfunctions we measure for 


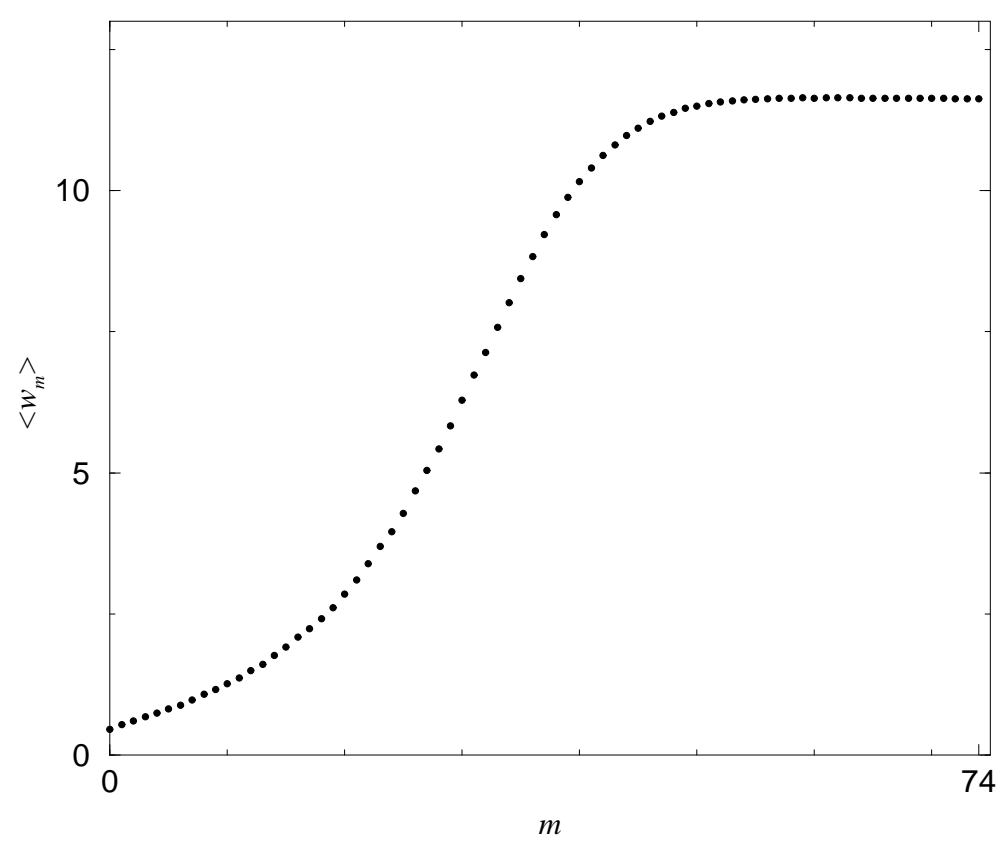

FIG. 6. Plot of $\bar{w}_{m}$ vs. $m$. We use a lattice of size $S=40$ with $\Delta=0.133$ and plot $\bar{w}_{m}$ for the first 75 eigenstates. We use 10000 samples and the parameters are the same as that of Fig. 2.

each eigenfunction the width defined by

$$
w_{m}^{2}=\int R^{2} \Phi_{m}^{2}(R) d R-\left(\int R \Phi_{m}^{2}(R) d R\right)^{2} .
$$

This width is closely related to the localization length $\ell_{m}$ defined earlier. We then average this quantity over many realizations of the random potential and in Fig. 6 we plot $\bar{w}_{m}$ vs. $m$. It is clear that as $m$ is increased the width of the eigenfunctions also increase. Clearly, on average the ground state is the state with the smallest width. This explains why the the distribution $P(\mathbf{R}, L)$ evolves with $L$ into fewer and fewer sharply localized peaks. This is just because the low energy eigenfunctions are sharply localized and they dominate the partition sum as $L$ is increased. The higher excited states have typically large localization lengths, and these are the states that dominate the partition sum when $L$ is short. For the lowest states we have verified the relation $w_{m}^{2} \sim 1 /\left|E_{m}\right|$. In Fig. 目 we plot $\bar{w}_{m}^{2}\left|\bar{E}_{m}\right|$ vs. $m$ for the lowest ten eigenfunctions and show that it is nearly constant in agreement with Eq. (3.6).

We now consider the role of the eigenfunctions on averaged quantities such as $\overline{\left\langle\mathbf{R}_{F}^{2}(L)\right\rangle}$ and $\overline{\left\langle\mathbf{R}_{Q}^{2}(L)\right\rangle}$. In order to study the effect of the eigenfunctions on $\overline{\left\langle\mathbf{R}_{F}^{2}(L)\right\rangle}$ we truncate 


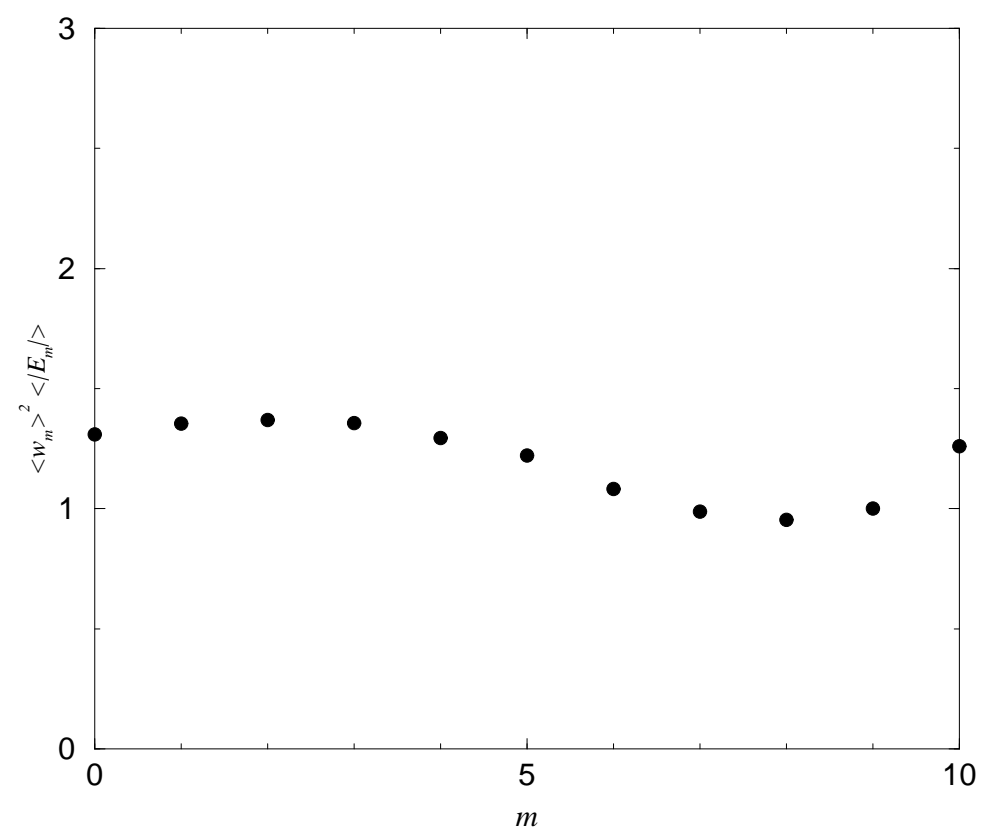

FIG. 7. Plot of $\bar{w}_{m}^{2}\left|\bar{E}_{m}\right|$ vs. $m$ for the lowest ten eigenfunctions. We average over 10000 samples and the parameters are the same as that of Fig. 2.

the sum over eigenstates $\sum_{0}^{N}$ to $\sum_{0}^{j}$ in the eigenfunction expansion in Eq. (3.9). So by changing the index $j$ we can see how the average end-to-end distance depends on how many eigenfunctions are kept in the expansion. In Fig. 8 we plot the average end-to-end distance $\overline{\left\langle\mathbf{R}_{F}^{2}(L)\right\rangle}$ for a number of different $j$ values. As expected the flat portion of the graph when $L$ is large is entirely due to the ground state contribution. So the constant $\overline{\left\langle\mathbf{R}_{F}^{2}(L)\right\rangle}$ is just a consequence of ground state dominance. This is consistent with the results in Sec. III where we showed that for long chains $\overline{\left\langle\mathbf{R}_{F}^{2}(L)\right\rangle}=2 d(d+1) \overline{\left(\ell_{g s}^{2}\right)}$ which is indeed independent of $L$. We can also see that the curved portion of the curve corresponds to the case before ground state dominance when a number of tail states contribute to the partition sum. For small $L$ we see that more than ten eigenfunctions are needed in order to capture the correct behavior of $\overline{\left\langle\mathbf{R}_{F}^{2}(L)\right\rangle}$.

As discussed in the previous paragraphs it is clear that the evolution of the partition sum is dependent on the nature of the eigenfunctions. Consequently, the sample-to-sample variation of quantities such as $\left\langle\mathbf{R}_{F}^{2}(L)\right\rangle_{V}$ and $\left\langle\mathbf{R}_{Q}^{2}(L)\right\rangle_{V}$ are also crucially dependent on the eigenfunctions. These sample-to-sample variations are important since they provide a 


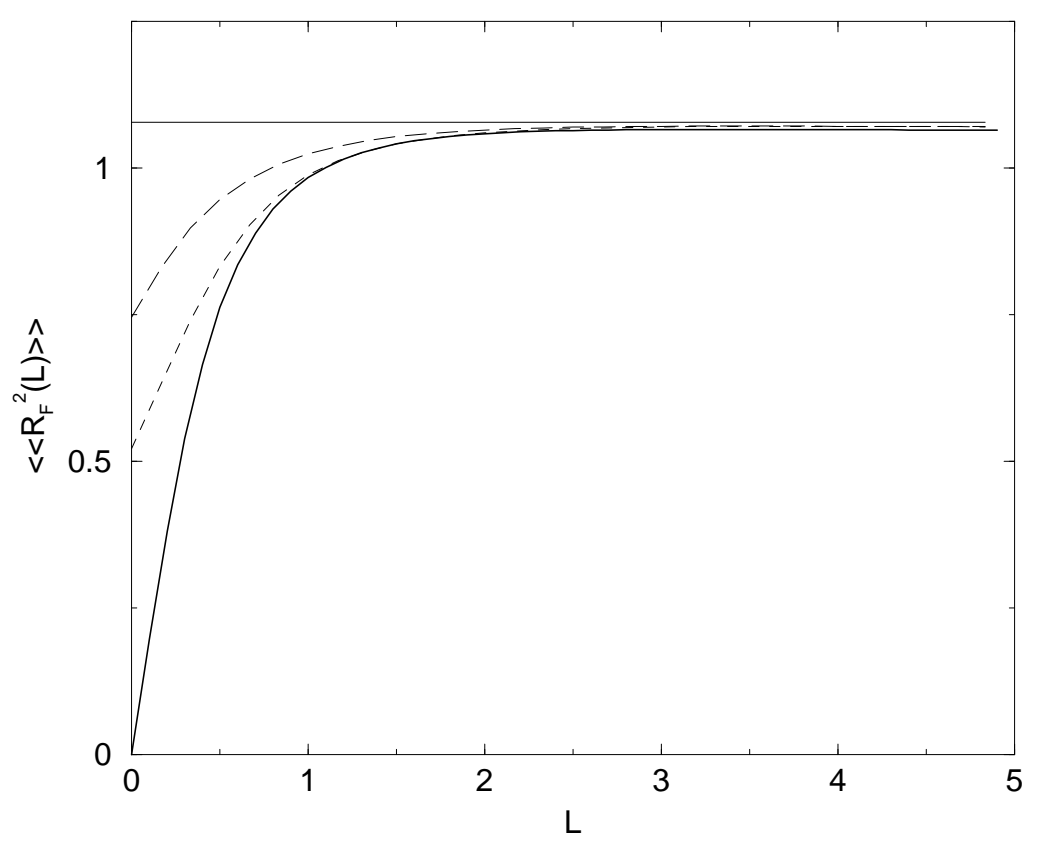

FIG. 8. Plot of $\overline{\left\langle\mathbf{R}_{F}^{2}(L)\right\rangle}$ vs. L. The thick solid line is computed using all the eigenfunctions. The thin solid line is found using only the ground state. The long dashed line is using the first five eigenfunctions and the short dashed line is using the first ten eigenfunctions.

measure of the glassiness of the system. In Fig. 9 we plot $\left\langle\mathbf{R}_{F}^{2}(L)\right\rangle_{V}$ vs. $L$ for 5 randomly picked samples. Notice that the spread of $\left\langle\mathbf{R}_{F}^{2}(L)\right\rangle_{V}$ around the average increases with $L$. In order to quantify this spread more accurately we plot the relative sample-to-sample fluctuations $\Delta_{F} / \overline{\left\langle\mathbf{R}_{F}^{2}(L)\right\rangle}$ vs. $L$ in Fig. 10. It is clear again that as the the length of the chain is increased the sample-to-sample fluctuations also increase. Comparing Fig. 10 with Fig. 8 it is clear that the sample to sample fluctuations increase rapidly at the same approximate value of $L$ at which the low energy eigenstates begin to dominate the behavior of $\overline{\left\langle\mathbf{R}_{F}^{2}(L)\right\rangle}$. The reason for this is that distributions dominated by localized states are strongly sample dependent. On the other hand when $L$ is small and the high excited states dominate, the sample-to-sample fluctuations are small since the shape of the extended states do not depend strongly on the random sample. So we can conclude that for a polymer in short range correlated random media, the glassy characteristics are directly related to the dominance of localized eigenfunctions. 


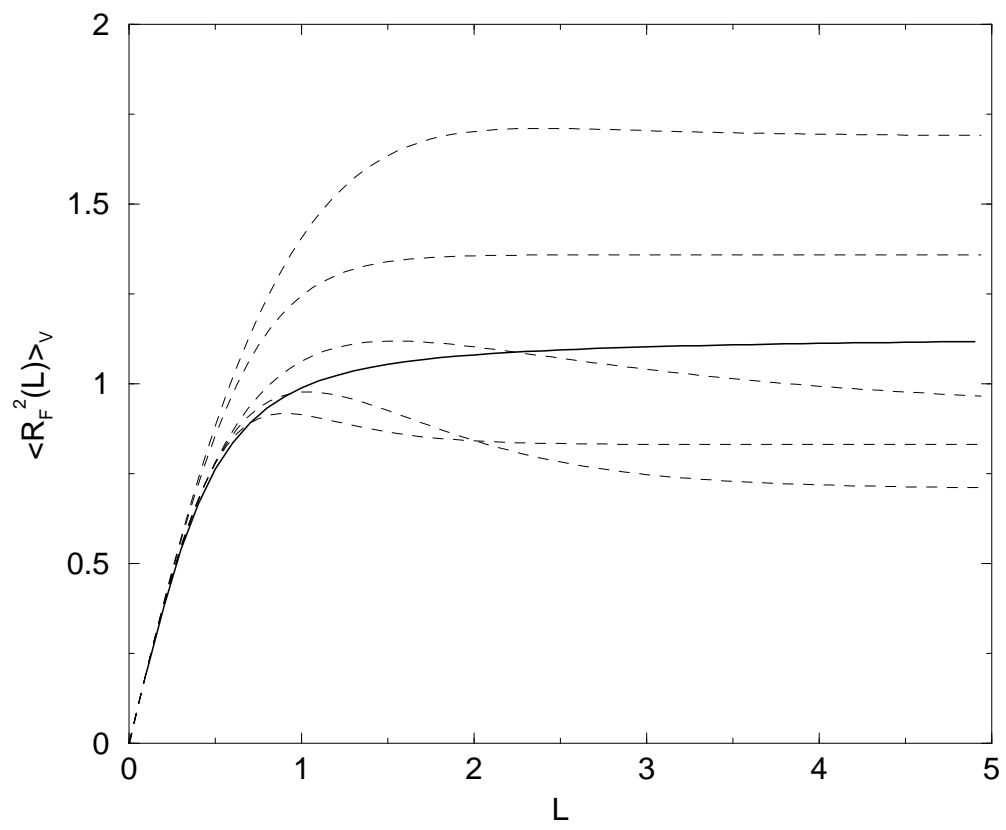

FIG. 9. Plot of $\left\langle\mathbf{R}_{F}^{2}(L)\right\rangle_{V}$ vs. $L$. The dashed lines correspond to five randomly chosen samples. The thick line is the average $\overline{\left\langle\mathbf{R}_{F}^{2}(L)\right\rangle}$ over 10000 samples.

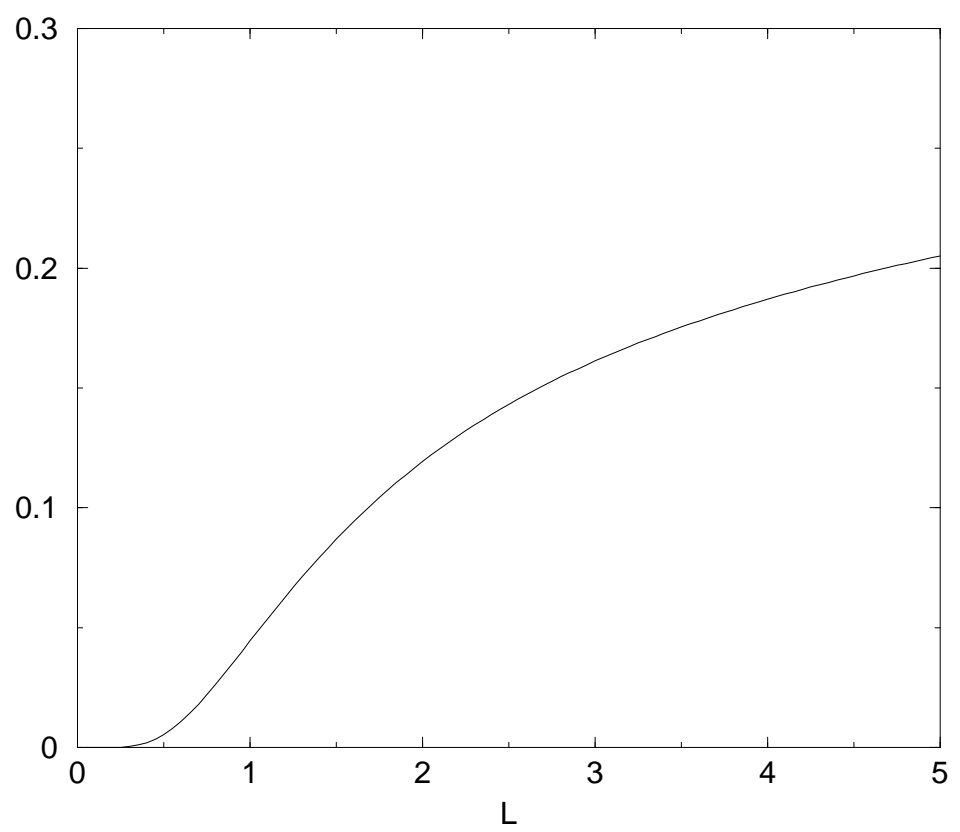

FIG. 10. Plot of $\Delta_{F} / \overline{\left\langle\mathbf{R}_{F}^{2}(L)\right\rangle}$ vs. L. The parameters are the same as in Fig. 2 and we average over 1000 samples. 


\section{Analysis of $\overline{\left\langle\mathbf{R}_{T}^{2}(L)\right\rangle}$}

We will now consider the end-to-end distance $\overline{\left\langle\mathbf{R}_{T}^{2}(L)\right\rangle}$. Since one end is tethered to the origin it turns out that this quantity behaves very differently from the quantities $\overline{\left\langle\mathbf{R}_{F}^{2}(L)\right\rangle}$ and $\overline{\left\langle\mathbf{R}_{Q}^{2}(L)\right\rangle}$. The replica variational approach described in Sec. $\mathbb{\mathbb { V }}$ is not adequate in this case in this case and hence we will only present the results of the lattice computation.

For short range correlations we found that the numerical method described in Sec. $\mathrm{V}$ was unreliable. The reason for this is that the sum over energy eigenfunctions in Eq. (3.3) is unstable since the overlap $\Phi_{m}(R) \Phi_{m}\left(R^{\prime}\right)$ (for short range correlations) is a number on the order of $\exp \left(-\left|R-R^{\prime}\right| / \ell_{m}\right)$ which is typically extremely small. However, we were able evaluate Eq. (3.3) accurately for small $\xi$ by solving the Schrödinger equation on a lattice using a fourth order Runge-Kutta algorithm with a very small time step $\left(t \sim 10^{-3}\right)$. We performed the computation on a large system $(S=160, N=800)$ in order to minimize finite size effects.

In Fig. 11 we plot $\overline{\left\langle\mathbf{R}_{T}^{2}(L)\right\rangle}$ vs. $L$ and also include $\left\langle\mathbf{R}_{T}(L)\right\rangle_{V}^{2}$ vs. $L$ for five typical samples. Notice that for a given sample the end-to-end distance $\left\langle\mathbf{R}_{T}(L)\right\rangle_{V}^{2}$ has a tendency to change rapidly and then remain constant. As discussed in Sec. III this is simply a consequence of hopping between localized states. That is, the free end of the polymer rapidly finds a deep well (which is also a localization center) and stays there. Upon averaging over 10000 random samples we find that $\overline{\left\langle\mathbf{R}_{T}^{2}(L)\right\rangle}$ grows linearly with $L$ for small $L$ but scales faster than diffusion for large $L$. The diffusive behavior when $L$ is small is not surprising since a short chain should not be affected by the random media. The claim based on Flory type arguments [4, 5] is that $\overline{\left\langle\mathbf{R}_{T}^{2}(L)\right\rangle} \sim L^{2} /(\log (L))^{\gamma}$ when $L$ is large. However, we were not able to extract a consistent exponent $\gamma$ for the range of $L$ that we explored. The reason for this is that our data is not accurate enough to detect the precise logarithmic correction to scaling.

However we went ahead and tried to find the best power law fit $\overline{\left\langle\mathbf{R}_{T}^{2}(L)\right\rangle} \propto L^{\nu}$ for large $L$. We found that for $5 \lesssim L \lesssim 25$ the exponent $\nu \approx 1.74$ yielded an excellent fit to the data. This result is consistent with the sub-ballistic prediction as it is very close but slightly less than ballistic scaling $\left(L^{2}\right)$. 


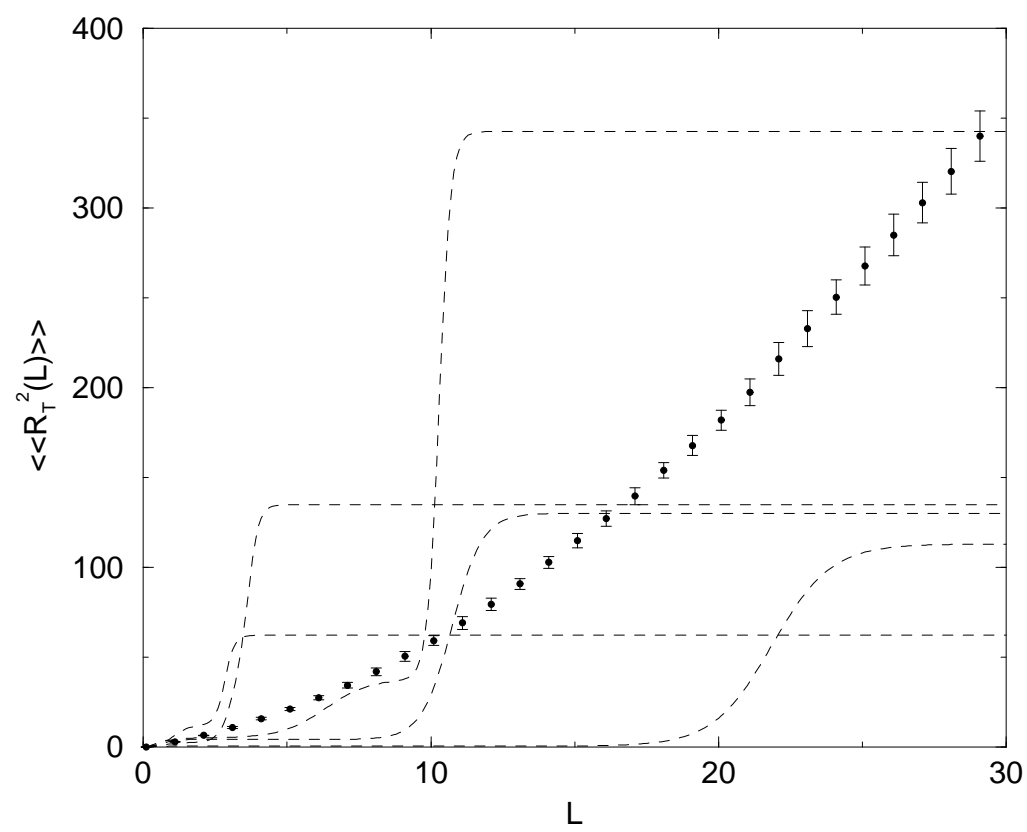

FIG. 11. Plot of $\overline{\left\langle\mathbf{R}_{T}^{2}(L)\right\rangle}$ vs. $L$. The full circles are generated by averaging over 10000 samples, and the error bars are found by computing the standard deviation of 10 sets of 1000 samples. The dashed lines are plots of $\left\langle\mathbf{R}_{T}(L)\right\rangle_{V}^{2}$ vs. $L$ for five typical samples. The parameters are the same as in Fig. 2, only that here the system size is four times larger $(S=160)$ and we set $\mu=0$. We use a lattice of 800 sites with $\Delta=0.2$. 


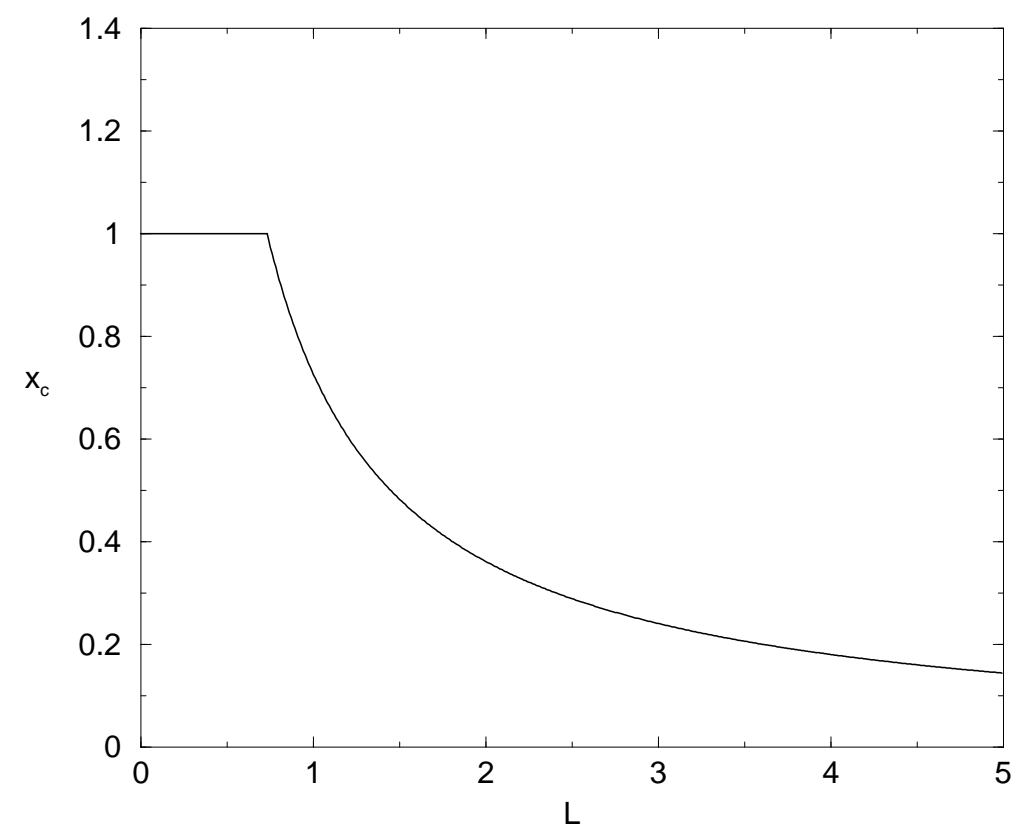

FIG. 12. Plot of $x_{c}$ vs. L. The parameters are the same as those used in Fig. 2.

\section{Analysis of the variational solution}

So far we have seen that the glassy characteristics of a polymer in a short range correlated random potential is closely related to the dominance of low energy eigenfunctions. We also know that the variational solution possesses a RS solution for $L<L_{c}$ and an RSB solution for $L>L_{c}$. It is well known that replica symmetry breaking is typically associated with glassy behavior, and for our model we show that the onset of RSB is precisely when the system begins to exhibit glassy behavior. The variational parameter that best reveals the transition between $\mathrm{RS}$ and $\mathrm{RSB}$ is the break point $x_{c}$. If $x_{c}=1$ then there is only an RS solution, and if $0<x_{c}<1$ then that corresponds to an RSB solution. In Fig. 12 we plot $x_{c}$ vs. $L$ using the same parameters that were used in Fig. 2. We can see that onset of the RSB solution is at $L_{c} \approx .73$. If we compare this result to the plot of $\Delta_{F} / \overline{\left\langle\mathbf{R}_{F}^{2}(L)\right\rangle}$ vs $L$ in Fig. 10, we see that near $L_{c} \approx 0.5$ the sample-to-sample fluctuations begin to rise rapidly. This result provides strong evidence that when the RSB solution is valid the polymer chain does indeed exhibit glassy behavior.

In Ref. [7] approximate analytic solutions to the variational equations were found. This 


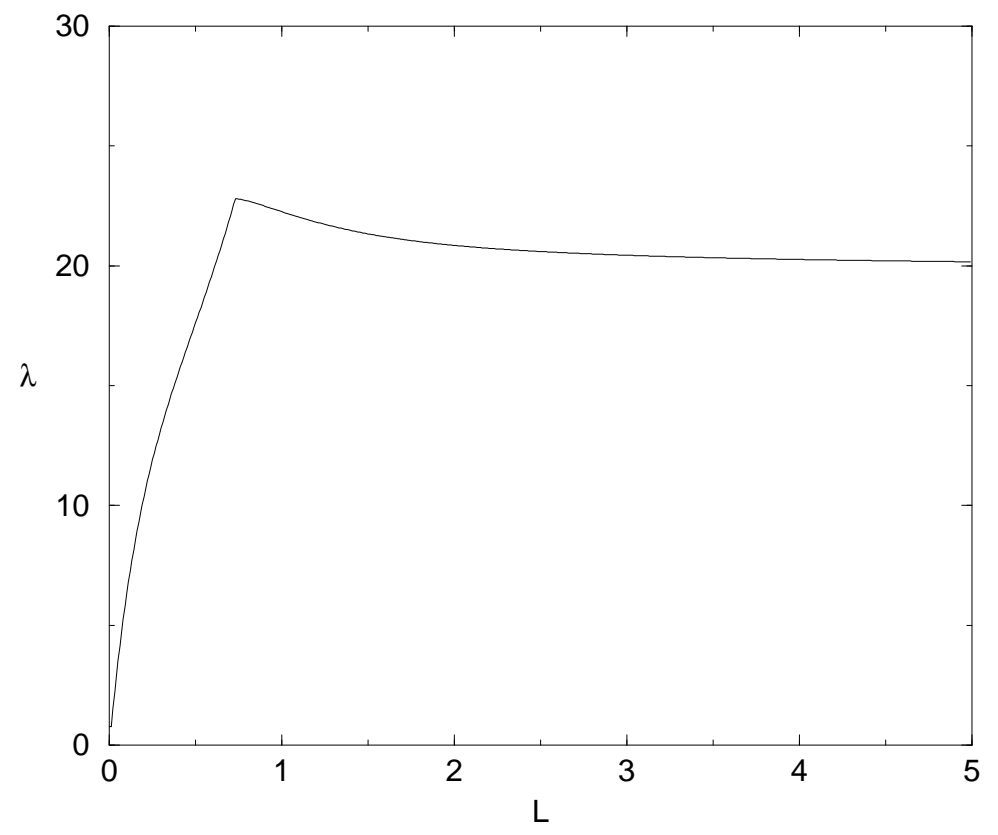

FIG. 13. Plot of $\lambda$ vs. L. The parameters are the same as those used in Fig. 2.

was for the case of large $L$, small $\mu$, and a delta correlated random potential $(\xi \rightarrow 0)$. It was found that

$$
x_{c}=\frac{1}{L}\left(\frac{d^{d-2}}{(2 \pi)^{d}} g^{2} \beta^{d+4} M^{d}|\log \mu|^{d-2}\right)^{-1 /(4-d)} .
$$

We checked the $L$ dependence of $x_{c}$ in Fig. 12 and indeed we found that $x_{c} \propto 1 / L$. In fact, we found that this $1 / L$ dependence is quite robust as it holds whenever there is a 1-step RSB solution. In a later section we will analyze the physical consequences of this behavior and show that it can be simply explained via the path integral mapping to the Schrödinger equation.

In Fig. 13 we plot the parameter $\lambda$ vs. $L$. The discontinuity is of course at $L_{c}$, after which we plot only the 1-step RSB solution. Notice that for $L>L_{c} \lambda$ is essentially constant, which by Eq. (4.9) implies that $\overline{\left\langle\mathbf{R}_{F}^{2}(L)\right\rangle}$ is also constant. This result is consistent with the approximate analytic solution in Ref. [7], where it was found that

$$
\lambda=\frac{d^{4 /(4-d)}}{(2 \pi)^{2 d /(4-d)}}\left(\beta^{2} M\right)^{(4+d) /(4-d)}(g|\log \mu|)^{4 /(4-d)},
$$

which is a constant independent of $L$. 


\section{PHYSICAL INTERPRETATION OF THE 1-STEP RSB SOLUTION}

In this section we study the physical interpretation of the replica symmetry breaking solution. Our purpose is to see if the underlying physical picture predicted by 1-step RSB is indeed consistent with the presence of exponentially localized eigenstates. The following analysis is valid for a very long polymer (large $L$ ) when the system becomes glassy. We begin by evaluating the replicated partition sum defined as

$$
\tilde{Z}_{n}\left(\left\{\mathbf{R}_{a}\right\}\right)=\int_{\mathbf{R}_{a}(0)=\mathbf{R}_{a}}^{\mathbf{R}_{a}(L)=\mathbf{R}_{a}} \prod_{a=1}^{n}\left[d \mathbf{R}_{a}\right] \exp \left(-\beta h_{n}\right),
$$

where $h_{n}$ is the quadratic trial Hamiltonian in Eq. (4.5). Since $h_{n}$ is quadratic the path integrals can be evaluated analytically and the final result can we written in the form

$$
\tilde{Z}_{n}\left(\left\{\mathbf{R}_{a}\right\}\right)=\text { const. } \times \exp \left(-\frac{1}{2} \sum_{a b} Q_{a b}^{-1} \mathbf{R}_{a} \cdot \mathbf{R}_{b}\right) .
$$

The details of this calculation along with the relationship between the matrices $Q_{a b}$ and $p_{a b}$ are given in the Appendix. Now, since $p_{a b}$ was parametrized according to the 1-step RSB scheme, it implies that $Q_{a b}$ can also be parametrized in the same way.

Mezard and Parisi [20] discuss the interpretation of a representation of the form (7.2) for the case of directed polymers. In particular they show how to deduce the structure of the probability distribution

$$
P_{V}(\mathbf{R})=\tilde{Z}_{V}(\mathbf{R}, L) / \int d \mathbf{R} \tilde{Z}_{V}(\mathbf{R}, L),
$$

which is the probability of finding a polymer loop that passes through $\mathbf{R}$ for a given re-

alization (which we denote by $V$ ) of the the random potential. Here $\tilde{Z}_{V}(\mathbf{R}, L)$ is just the partition sum $Z(\mathbf{R}, \mathbf{R} ; L)$ as given in Eq. (2.4). This probability is related to the replicated partition function given in Eq. (7.1) by

$$
P_{V}(\mathbf{R})=\lim _{n \rightarrow 0} \int d \mathbf{R}_{2} \cdots d \mathbf{R}_{n} \tilde{Z}_{n}\left(\left\{\mathbf{R}_{a}\right\}\right)_{\mathbf{R}_{1}=\mathbf{R}} .
$$

Mezard and Parisi's analysis has to be adapted for the case of real (non-directed) polymers of length $L$ in a random potential which is independent of time. The changes will be pointed out below. 
If $Q_{a b}$ is parametrized by $\{\tilde{q}, q(x)\}$ such that

$$
q(x)= \begin{cases}q_{0} & x<x_{c} \\ q_{1} & x>x_{c}\end{cases}
$$

one proceeds to obtain $P_{V}(\mathbf{R})$ by the following procedure:

1. For each sample ( a realization of the random potential) generate a random variable $\mathbf{R}_{0}$ which is picked from the distribution

$$
\mathcal{P}\left(\mathbf{R}_{0}\right)=\frac{1}{\left(2 \pi q_{0}\right)^{d / 2}} \exp \left(-\frac{\mathbf{R}_{0}^{2}}{2 q_{0}}\right)
$$

2. Consider a set of "states" labeled by the index $\alpha$ whose physical meaning will be elucidated shortly. Each of these states is characterized by a weight $W_{\alpha}$ and a position variable $\mathbf{R}_{\alpha}$. Given $\mathbf{R}_{0}$, the variables $\mathbf{R}_{\alpha}$ are an infinite set of uncorrelated random variables distributed according to

$$
\mathcal{P}\left(\mathbf{R}_{1}, \mathbf{R}_{2}, \cdots\right)=\prod_{\alpha} \frac{1}{\left(2 \pi\left(q_{1}-q_{0}\right)\right)^{d / 2}} \exp \left(-\frac{\left(\mathbf{R}_{\alpha}-\mathbf{R}_{0}\right)^{2}}{2\left(q_{1}-q_{0}\right)}\right) .
$$

The distribution of weights will be discussed below.

3. Given these "states" for a given sample, The probability distribution $P_{V}(\mathbf{R})$ for that sample has the form

$$
P_{V}(\mathbf{R})=\sum_{\alpha} W_{\alpha} \frac{1}{\left(2 \pi\left(\tilde{q}-q_{1}\right)\right)^{d / 2}} \exp \left(-\frac{\left(\mathbf{R}-\mathbf{R}_{\alpha}\right)^{2}}{2\left(\tilde{q}-q_{1}\right)}\right) .
$$

The weights $W_{\alpha}$ are given in terms of some "free energy" variables $f_{\alpha}$ :

$$
W_{\alpha}=\frac{\exp \left(-\beta f_{\alpha}\right)}{\sum_{\gamma} \exp \left(-\beta f_{\gamma}\right)}
$$

These free energy variables are chosen from an exponential distribution

$$
P\left[f_{\alpha}\right] \propto \exp \left(x_{c} \beta f_{\alpha}\right) \theta(f-\bar{f})
$$

where $\bar{f}$ is an upper cutoff. 
What is the meaning of these variables in the present case? To determine the weights $W_{\alpha}$ we compare Eq. (7.8) to the eigenfunction expansion given in Eq. (3.7). From Eq. (3.7) together with Eq. (7.3) it becomes clear that

$$
P_{V}(\mathbf{R})=\sum_{\alpha} A_{\alpha}\left|\Phi_{\alpha}(\mathbf{R})\right|^{2}
$$

where

$$
A_{\alpha}=\frac{\exp \left(-\beta L E_{\alpha}\right)}{\sum_{\gamma} \exp \left(-\beta L E_{\gamma}\right)}
$$

Comparing Eq. (7.8) and Eq. (7.12) it becomes obvious that

$$
W_{\alpha}=A_{\alpha},
$$

and

$$
\Phi_{\alpha}^{2}(\mathbf{R}) \propto \exp \left(-\frac{\left(\mathbf{R}-\mathbf{R}_{\alpha}\right)^{2}}{2\left(\tilde{q}-q_{1}\right)}\right) .
$$

Hence, the "states" labeled by $\alpha$ are in our case the actual eigenstates of the imaginary time Schrödinger equation (3.4). These are localized tail states centered at position $\mathbf{R}_{\alpha}$ with an associated "weight" $W_{\alpha}$. Thus the 1-step RSB solution approximates the tail states by a fixed Gaussian form. The width of these Gaussians $\left(w_{0}\right)$ as defined by Eq. (6.1) satisfies $w_{0}^{2}=d\left(\tilde{q}-q_{1}\right)$, which for large $L$ can be shown to converge to $w_{0}^{2} \sim d /(2 \beta \sqrt{\lambda M}$ ) (see Appendix). Since $\lambda$ becomes constant for large $L$ then so does the width $w_{0}$. Thus the 1-step RSB solution approximates all the localized states by a Gaussian of constant width $w_{0}$, which is the typical size of a Gaussian chain embedded in a random potential. It also becomes evident that the free energies $f_{\alpha}$ are equal to $L E_{\alpha}$. This make sense if we think of $\left|E_{\alpha}\right|$ as representing the binding energy per monomer, and thus $f_{\alpha}=L E_{\alpha}$ represent the total energy of the chain. Alternatively one can start with the quantum particle picture and identify $f_{\alpha}$ with $E_{\alpha}$ but then in the transformation from a quantum particle to a polymer one has to replace $\beta$ by $\beta L$, and thus one ends up with the same expressions.

These arguments lead us to expect that within the 1-step RSB scheme, the energy variables $E_{\alpha}$ are independent random variables taken from an exponential distribution:

$$
P\left[E_{\alpha}\right] \propto e^{\beta L x_{c} E_{\alpha}} \theta\left(\bar{E}-E_{\alpha}\right)
$$


with $\bar{E}$ being some energy scale determined by the upper cutoff of the tail region. We will now argue that the distribution given above is just the expected distribution of ground-state energies i.e. the probability of finding the lowest energy level to have energy $E$. We first review some very basic results of extreme value statistics as presented in Ref. [24]. Given $K$ independent and identically distributed random variables $E_{i}$, pulled from a distribution of the form

$$
\tilde{P}(E)=\frac{A}{|E|^{\alpha}} \exp \left(-B|E|^{\delta}\right)
$$

the probability that the lowest of the $K$ energies is $E$ (for $E \rightarrow-\infty$ and $K \rightarrow \infty$ ) is given by

$$
P(E) \propto \exp \left[B \delta\left|E_{c}\right|^{\delta-1} E\right]
$$

where

$$
E_{c}=-\left(\frac{\log (K)}{B}\right)^{1 / \delta}
$$

The value of $E_{c}$, the lowest energy expected to be attained in $\mathrm{K}$ trials, is easily obtained from

$$
\int_{-\infty}^{E_{c}} d E \tilde{P}(E) \simeq 1 / K
$$

The reason why we chose a distribution of the form given in Eq. (7.16) is that in $d=1$ the probability $\tilde{P}(E)$ is known to have that form exactly for the case of delta correlated random potentials (see [18]). For $d>1$ Lifshits [25] argued that the form given by Eq. (7.16) is also valid. Our goal now is to see if the distribution Eq. (7.15) derived using the 1-step RSB solution is indeed consistent with the distribution Eq. (7.17) predicted using extreme value statistics.

Comparing Eq. (7.15) and Eq. (7.17) we find that for consistency the break point should satisfy

$$
x_{c}=\frac{\delta}{\beta L} B^{1 / \delta}(\log (K))^{(\delta-1) / \delta} .
$$


Notice that the $1 / L$ behavior of $x_{c}$ is exactly the same as was found analytically for large $L$ in Ref. [7] and numerically for any $L>L_{c}$ in the present work.

We can go further by using the fact that the number of energy levels $K$, within a fixed energy interval is directly proportional to the system size, which in our formulation is effectively determined by $\mu$. Assuming $\log (K) \propto|\log (\mu)|$ and comparing to the approximate solution for $x_{c}$ in Eq. (6.2) we find that $\delta=(4-d) / 2$ and $B \propto 1 / g$. Now $\tilde{P}(E)$ is just proportional to the density of states $\rho(E)$, and it is known exactly in one dimension. Indeed, when $d=1, \delta=3 / 2$ and $B \propto 1 / g$. For $2 \leq d<4, \delta$ agrees with the result derived by Lifshits [25]. Hence, the exponent $\delta$ and the disorder dependence of $B$ is correctly predicted by the 1-step RSB solution. The exponent $\alpha$ cannot be found since the statistics of the lowest energy depends only on the exponential tail.

The 1-step RSB solution is thus in agreement with Eq. (7.17). Since $x_{c} \propto 1 / L$ then the distribution of the energies predicted from Eq. (7.15) is independent of $L$. Note also that the value of $E_{c}$ which gives a typical lowest energy for a given volume of the system coincides with the expected value given in Eq. (2.7) when one substitutes for $B \sim 1 / g, \delta=(4-d) / 2$ and $\ln K \sim \ln \mathcal{V}$ in Eq. (7.18). The localization length which is the width of a localized state is known to be related to the energy of the state by

$$
\ell \sim \frac{1}{\beta|2 M E|^{1 / 2}} .
$$

If we use $E_{c}$ for the value of the lowest energy in a volume $\mathcal{V}$ we get an estimate for $R_{F}$, the radius of gyration of a free chain which is in agreement with Eq. (2.6).

When $L \rightarrow \infty$ most of the weights $W_{\alpha} \rightarrow 0$ and only the weight with the lowest $E_{\alpha}$ will contribute significantly to the distribution $P_{V}(\mathbf{R})$. So in this limit $P_{V}(\mathbf{R})$ will be approximated by a single Gaussian located somewhere in the sample. This result is consistent with ground-state dominance as predicted by the corresponding eigenfunction expansion.

We now consider the distribution $P\left(\mathbf{R}_{\alpha}\right)$ given in Eq. (7.7). This is just the distribution for the localization centers $\mathbf{R}_{\alpha}$ for a given value of $\mathbf{R}_{0}$. Hence, we can calculate the average distance between the localized states for a given sample. We find that the width $w$ of the Gaussian $P\left(\mathbf{R}_{\alpha}\right)$ satisfies $w^{2}=d\left(q_{1}-q_{0}\right)$. For small $\mu$ and large $L$ we find (see Appendix) 
that $w^{2} \approx d /\left(\beta \mu L x_{c}\right)$. Using the analytic approximation for $x_{c}$ we find that

$$
w^{2} \sim \frac{1}{\mu} g^{2 /(4-d)}|\log (\mu)|^{(d-2) /(4-d)} .
$$

We can also find the average distance from the origin to a localization center $\mathbf{R}_{\alpha}$. To do this we have to compute the average probability of find a localization center at $\mathbf{R}_{\alpha}$. This is

$$
\begin{array}{r}
\bar{P}\left(\mathbf{R}_{\alpha}\right) \propto \int d \mathbf{R}_{0} \exp \left(-\mathbf{R}_{0}^{2} /\left(2 q_{0}\right)\right) \exp \left(-\left(\mathbf{R}_{\alpha}-\mathbf{R}_{0}\right)^{2} /\left(2\left(q_{1}-q_{0}\right)\right)\right. \\
=\exp \left(-\mathbf{R}_{\alpha}^{2} /\left(2 q_{1}\right)\right)
\end{array}
$$

If we estimate the average distance to a localization center to be the width of the Gaussian $\bar{P}\left(\mathbf{R}_{\alpha}\right)$, then $w^{2}=d q_{1} \approx d /\left(\beta \mu L x_{c}\right)$ which is the same as the average distance $w$ between tail states derived above. This is because the fluctuations of $\mathbf{R}_{0}$ are small compared to the fluctuations of $\mathbf{R}_{\alpha}-\mathbf{R}_{0}$ when the volume is large. Notice that this result is consistent with our calculation for $\overline{\left\langle\mathbf{R}_{Q}^{2}(L)\right\rangle}$ in Eq. (4.10), since for $L$ large $\overline{\left\langle\mathbf{R}_{Q}^{2}(L)\right\rangle} \sim d /\left(\beta \mu L x_{c}\right)$. These quantities should be close since both give an approximation to the average distance to the ground state localization center.

So far we have studied the physical interpretation of the 1-step RSB solution, but recall that for $L<L_{c}$ there was only a RS solution. It is interesting to study the physical implications of this RS solution. In this case the probability distribution $P_{V}(\mathbf{R})$ will have the simple form

$$
P_{V}(\mathbf{R})=\frac{1}{\left(2 \pi\left(\tilde{q}-q_{0}\right)\right)^{d / 2}} \exp \left(-\frac{\left(\mathbf{R}-\mathbf{R}_{0}\right)^{2}}{2\left(\tilde{q}-q_{0}\right)}\right)
$$

where the variable $\mathbf{R}_{0}$ is taken from the distribution

$$
\mathcal{P}\left(\mathbf{R}_{0}\right)=\frac{1}{\left(2 \pi q_{0}\right)^{d / 2}} \exp \left(-\frac{\mathbf{R}_{0}^{2}}{2 q_{0}}\right)
$$

Notice that for a given sample $P_{V}(\mathbf{R})$ is approximated by a Gaussian shifted from the origin by a distance $\mathbf{R}_{0}$. The width of this Gaussian can be shown to be essentially independent of disorder in the limit $\mu \rightarrow 0$, as the width $w_{0}$ satisfies $w_{0}^{2} \sim d(\beta \mu L)^{-1}$, which is the result for the case of zero disorder. The average shift from the origin can be estimated by the width $w_{1}$ of the Gaussian in Eq. (7.25), which satisfies $w_{1}^{2} \sim g \beta^{(d+2) / 2} L^{(d+2) / 2} \mu^{(d-2) / 2}$, for the case 
of delta correlations and large volume $(\mu \rightarrow 0)$. This result is similar to that found for the case of long range correlated random media in which it was found that the only effect of disorder is to shift the center of mass of the polymer chain from the origin.

We can conclude that the physical interpretation of the 1-step RSB solution is consistent with that given by the eigenfunction expansion. Clearly, the 1-step RSB solution captures the localized states and also correctly predicts some important features of the eigenvalue distribution. However, there are differences and these reveal the limitations of the 1-step RSB solution. For example, all the localized states are approximated by the same Gaussian profile when in fact the localization lengths should increase with energy.

\section{CONCLUDING REMARKS}

The findings in this paper allow us to give a fairly complete description of a polymer in random medium. We find that the size of a short polymer chain behaves as if there is no disorder, but that the position of the center of mass of the chain is strongly affected by the random media. Applying the replica variational method we find that short chains are well described by a RS solution. Also, using an eigenfunction expansion we show that the partition sum of short chains is dominated by extended eigenstates. For long chains

the physical picture becomes more interesting and complex. We find that long chains are likely to be localized in regions of the sample where there is very low potential energy. These regions correspond to localized tail states of the corresponding Schrödinger equation. As the length of the chain increases the number of dominant conformations decreases until finally the chain is essentially localized in one small region, consistent with ground state dominance. In terms of the mapping from a chain to a quantum particle, a big chain length corresponds to a very low temperature for the quantum particle.

We show that the onset of the variational RSB solution to the stationarity equations corresponds to the dominance of localized tail states. This 1-step RSB solution correctly describes the glassy characteristics of the polymer chain such as large sample-to-sample variations. We have demonstrated the clear physical picture associated with the abstract 


\section{RSB solution.}

Our analysis also suggests a direct connection between the size of a polymer in a random potential with short range correlations, and the localization length associated with the lowest energy state in a system of finite volume $\mathcal{V}$. Given the density of states $\rho(E)$ we can estimate the lowest energy $E_{c}$ by

$$
\int_{-\infty}^{E_{c}} d E \rho(E) \simeq 1 / \mathcal{V}
$$

The size of a free Gaussian chain, in the long chain limit, is then given by

$$
R_{F} \sim\left|E_{c}\right|^{-1 / 2}
$$

It is remarkable that starting from an annealed average (the density of states involves the average of the partition function) and using extreme value statistics plus the known relationship between the localization length and the value of the tail state energy, we can predict the behavior of a quenched average like the chain size.

It is well known that glassy systems are notoriously difficult to simulate using the Monte Carlo method since equilibration times are exceedingly large. In the context of a polymer chain in a random medium, we expect that a long chain will get trapped in the deep wells of the random potential and any local updating procedure, such as the Metropolis scheme, will not be able to find the true minimum energy configurations in a reasonable time. A workaround is to use a simulated annealing procedure [15]. Of course, these traps are just those regions of the sample where an eigenstate would be localized. They correspond to localized states with energies above the true ground state that exists for a finite-size system. Since the partition sum for a long chain is dominated by localized tail states those chain configurations that are in the vicinity of such a state are overwhelmingly more favorable than other configurations. All the classic characteristics of a glassy system emerge (like trapping, metastability, aging), and they have a clear physical origin in terms of the localization picture. 


\section{ACKNOWLEDGMENTS}

This research is supported by the US Department of Energy (DOE), grant No. DE-G02-

98ER45686. Y. Y. G. thanks the Weizmann Institute for a Meyerhoff Visiting Professorship during which some of this reseach has been done. 


\section{APPENDIX:}

In this appendix we derive the relation between the matrix $p_{a b}$ (see Eq. (4.7)) characterizing the variational hamiltonian and the matrix $Q_{a b}$ defined in Eq. (7.2). We start from

$$
Z_{n}\left(\left\{\mathbf{R}_{a}^{\prime}\right\},\left\{\mathbf{R}_{a}\right\} ; L\right)=\int_{\mathbf{R}_{a}(0)=\mathbf{R}_{a}^{\prime}}^{\mathbf{R}_{a}(L)=\mathbf{R}_{a}} \prod_{a=1}^{n}\left[d \mathbf{R}_{a}(u)\right] \exp \left(-\beta h_{n}\right)
$$

with

$$
\begin{aligned}
h_{n}= & \frac{1}{2} \int_{0}^{L} d u \sum_{a}\left[M\left(\frac{d \mathbf{R}_{a}(u)}{d u}\right)^{2}+\lambda \mathbf{R}_{a}^{2}(u)\right] \\
& +\frac{1}{2 L} \int_{0}^{L} d u \int_{0}^{L} d u^{\prime} \sum_{a b} p_{a b} \mathbf{R}_{a}(u) \cdot \mathbf{R}_{b}\left(u^{\prime}\right) .
\end{aligned}
$$

Let $O_{a b}$ be an orthogonal matrix which diagonalizes $p_{a b}$ by a similarity transformation, and let $p_{c}, c=1, \cdots, n$ be the eigenvalues of $p_{a b}$. We can express $Z_{n}$ in the form

$$
Z_{n}=\int \prod_{c} d \boldsymbol{\lambda}_{c} \exp \left(-\sum_{c} \frac{\boldsymbol{\lambda}_{c}}{2}\right) \int_{\mathbf{R}_{a}(0)=\mathbf{R}_{a}^{\prime}}^{\mathbf{R}_{a}(L)=\mathbf{R}_{a}} \prod_{a=1}^{n}\left[d \mathbf{R}_{a}(u)\right] \exp \left(-\beta h_{n}\left(\left\{\boldsymbol{\lambda}_{c}\right\}\right)\right),
$$

with

$h_{n}\left(\left\{\boldsymbol{\lambda}_{c}\right\}\right)=\frac{1}{2} \int_{0}^{L} d u \sum_{a}\left[M\left(\frac{d \mathbf{R}_{a}(u)}{d u}\right)^{2}+\lambda \mathbf{R}_{a}^{2}(u)+\sum_{c} \sqrt{-p_{c} /(\beta L)} O_{a c} \boldsymbol{\lambda}_{c} \cdot \mathbf{R}_{a}(u).\right]$

The path integral can now be carried out using Eqns. (3.39-3.41) in [16]. The result is

$$
Z_{n}=\int \prod_{c} d \boldsymbol{\lambda}_{c} \exp \left(-\sum_{c} \frac{\boldsymbol{\lambda}_{c}}{2}\right) e^{-\beta \Phi}
$$

where

$\Phi=A\left(\sum \mathbf{R}_{a}^{2}+\sum \mathbf{R}_{a}^{\prime 2}\right)+B\left(\sum \mathbf{R}_{a}+\sum \mathbf{R}_{a}^{\prime}\right) \cdot \mathbf{V}_{a}+2 C \sum \mathbf{R}_{a} \cdot \mathbf{R}_{a}^{\prime}+D \sum \mathbf{V}_{a}^{2}$,

with

$$
\begin{aligned}
A & =\frac{1}{2} \sqrt{\lambda M} \operatorname{coth}(L \sqrt{\lambda / M}) \\
B & =\sqrt{\lambda M}[\cosh (L \sqrt{\lambda / M})-1][\sinh (L \sqrt{\lambda / M})]^{-1} \\
C & =-\frac{1}{2} \sqrt{\lambda M}\left[(\sinh (L \sqrt{\lambda / M})]^{-1}\right. \\
D & =B-L \lambda / 2 \\
\mathbf{V}_{a} & =\frac{1}{\lambda} \sum_{c} \sqrt{-p_{c} /(\beta L)} O_{a c} \boldsymbol{\lambda}_{c} .
\end{aligned}
$$


Notice that

$$
\sum_{a} \mathbf{V}_{a}^{2}=-\frac{1}{\lambda^{2} \beta L} \sum_{a} p_{a} \boldsymbol{\lambda}_{a}^{2}
$$

We now put $\mathbf{R}_{a}=\mathbf{R}_{a}^{\prime}$ and integrate out over $\boldsymbol{\lambda}_{c}$ to obtain

$$
Z_{n}\left(\left\{\mathbf{R}_{a}\right\},\left\{\mathbf{R}_{a}\right\} ; L\right)=\mathcal{N} \exp \left(-2 \beta \sum_{a b}\left[(A+C) \delta_{a b}-\beta B^{2} \mathcal{M}_{a b}\right] \mathbf{R}_{a} \cdot \mathbf{R}_{b}\right)
$$

with

$$
\mathcal{M}=\frac{1}{2 \beta D}\left(\mathbf{1}-\frac{\lambda^{2} L}{2 D} \mathbf{p}^{-1}\right)^{-1}
$$

Here we denoted by $\mathbf{1}$ the unit $n \times n$ matrix, and $\mathbf{p}$ stands for the matrix $p_{a b}$. Comparing with Eq. (7.2) we find that

$$
Q_{a b}=\frac{1}{4 \beta}\left\{\left[(A+C) \mathbf{1}-\beta B^{2} \mathcal{M}\right]^{-1}\right\}_{a b}
$$

In the Appendix of Ref. [20] one can find a formula for the inverse of a hierarchical matrix. For the one-step RSB case, the inverse of a matrix $\mathbf{p}=\left\{\tilde{p}, p_{0}, p_{1}\right\}$ is given by $\mathbf{q}=\mathbf{p}^{-1}$ where

$$
\begin{aligned}
\tilde{q} & =\frac{1}{\left(\tilde{p}-p_{0} x_{c}-p_{1}\left(1-x_{c}\right)\right)}\left(1-\frac{p_{0}}{\tilde{p}-p_{0} x_{c}-p_{1}\left(1-x_{c}\right)}-\frac{\left(p_{1}-p_{0}\right)\left(1-x_{c}\right)}{\tilde{p}-p_{1}}\right), \\
q_{0} & =-\frac{p_{0}}{\left(\tilde{p}-p_{0} x_{c}-p_{1}\left(1-x_{c}\right)\right)^{2}}, \\
q_{1} & =-\frac{1}{\tilde{p}-p_{0} x_{c}-p_{1}\left(1-x_{c}\right)}\left(\frac{p_{1}-p_{0}}{\tilde{p}-p_{1}}+\frac{p_{0}}{\tilde{p}-p_{0} x_{c}-p_{1}\left(1-x_{c}\right)}\right) .
\end{aligned}
$$

In the limit of large $L$ we have

$$
\begin{array}{r}
A \rightarrow \frac{1}{2} \sqrt{\lambda M} \\
B \rightarrow \sqrt{\lambda M} \\
C \rightarrow 0 \\
D \rightarrow-\frac{L \lambda}{2}+\sqrt{\lambda M} .
\end{array}
$$

In addition we use the one step breaking results found in Ref. [7]. Evaluating the matrix $Q$ in the limit of small $\mu$ we find to leading order 


$$
\begin{aligned}
\tilde{q} & =\frac{1}{L x_{c} \beta \mu}+\frac{s_{0}}{L \beta \mu^{2}}+\frac{1}{2 \beta \sqrt{M \lambda}}+\cdots \\
q_{0} & =\frac{s_{0}}{L \beta \mu^{2}}+\cdots \\
q_{1} & =\frac{1}{L x_{c} \beta \mu}+\frac{s_{0}}{L \beta \mu^{2}}+\cdots
\end{aligned}
$$

with the parameters $\lambda$, and $x_{c}$ given in Eq. (4.13) and (4.14) respectively and $s_{0}$ is given approximately by [0]

$$
s_{0}=\text { const. } \times g^{(2-d) / 4} \beta L(\beta \sqrt{M \lambda})^{-d(d+2) / 8} \mu^{d / 2+1}|\ln \mu|^{(d+2) / 4} .
$$

We have also used the fact that $\Sigma=\lambda$ in this limit. 


\section{REFERENCES}

[1] A. Baumgartner and M. Muthukumar, J. Chem. Phys. 87, 3082 (1987).

[2] S. F. Edwards and M. Muthukumar, J. Chem. Phys. 89, 2435 (1988).

[3] M. E. Cates and C. Ball, J. Phys. (France) 89, 2435 (1988).

[4] T. Nattermann and W. Renz, Phys. Rev. A 40, 4675 (1989).

[5] Y. C. Zhang, Phys. Rev. Lett. 56, 2113 (1986).

[6] A. Baumgartner and M. Muthukumar in Advances in Chemical Physics (vol. XCIV) Polymeric Systems I. Prigogine and S. A. Rice editors, (John Wiley \& Sons, Inc., New York, 1996).

[7] Y. Y. Goldschmidt, Phys. Rev. E 61, 1729 (2000).

[8] Y. Shiferaw and Y. Y. Goldschmidt J. Phys. A: Math. Gen. 33, 4461 (2000).

[9] Y. Y. Goldschmidt, Phys. Rev. E 53, 343 (1996).

[10] D. R. Nelson and V. M. Vinokur, Phys. Rev. B 48, 13060 (1993).

[11] Y. Y. Goldschmidt, Phys. Rev. B 56, 2800 (1997).

[12] P. W. Anderson, Phys. Rev. bf 109, 1492 (1958).

[13] B. Souillard, in Chance and Matter, Proceedings of the Les Houches Lectures, 1986, Eds. J. Souletie, J. Vannismenus, and R. Stora (North-Holand, Amsterdam, 1987)

[14] M. Doi and S. F. Edwards, The Theory of Polymer Dynamics (Oxford University Press, Oxford, 1986).

[15] H-Y. Chen and Y. Y. Goldschmidt, J. Phys. A: Math. Gen. 30, 1803 (1997).

[16] R. P. Feynman, Statistical Mechanics: A Set of Lectures (Benjamin, New York, 1972).

[17] J. Fröhlich and T. Spencer, Commun. Math. Phys. 88, 151 (1983). 
[18] B. Halperin, Phys. Rev. 139, A104 (1965).

[19] E. I. Shakhnovich and A. M. Gutin, J. Phys. A 22, 1647, (1989).

[20] M. Mezard and G. Parisi, J. Phys. I France 1, 809 (1991).

[21] M. Mezard, G. Parisi and M. A. Virasoro, Spin glass theory and beyond (World Scientific, Singapore, 1987).

[22] W. H. Press et al., Numerical Recipes in Fortran, 2nd ed. (Cambridge University Press 1992).

[23] H. A. Makse, S. Havlin, H. E. Stanley and M. Schwartz, CHAOS SOLITON FRACT 6, 295 (1995).

[24] J. P. Bouchaud and M. Mezard, J. Phys. A: Math. Gen, 30 (1997) 7997.

[25] I. M. Lifshitz, S.A. Gredeskul, and L.A. Patur, Introduction to the Theory of Disordered Systems (Wiley, NY, 1988); I. M. Lifshits, adv. Phys. 13, 483 (1964).

[26] J. Zittartz and J.S. Langer, Phys. Rev. 148741 (1966). 